\title{
Vibrational and Chemical Kinetics in Non-Equilibrium Gas Flows
}

\author{
E.V. Kustova and E.A. Nagnibeda \\ Saint Petersburg State University \\ Russia
}

\section{Introduction}

In this chapter, we consider the vibrational and chemical kinetics in reacting gas flows under the conditions of strong deviations from thermodynamic equilibrium. Such conditions occur, for example, near surfaces of nonexpendable space vehicles in their reentry into the Earth and Mars atmospheres, in experiments carried out in high-enthalpy facilities, in supersonic gas flows in nozzles and jets, in chemical technology processes. In many cases, the characteristic times of vibrational relaxation and chemical reactions appear to be comparable with the characteristic time for the variation of basic gas-dynamic parameters of a flow. Therefore, the equations of gas dynamics and non-equilibrium kinetics should be considered jointly. Consequently, the set of governing equations for macroscopic parameters includes not only the conservation equations for the momentum and total energy, but also the equations for chemical reactions and vibrational energy relaxation. The latter equations contain the rates of energy transitions and chemical reactions which are needed in order to solve the equations of non-equilibrium gas flows.

Originally, non-equilibrium chemical reactions were studied in thermally equilibrium gas mixtures which were assumed to be spatially homogeneous Kondratiev \& Nikitin (1974). Later on, different models for vibrational-chemical coupling were proposed on the basis of the kinetic theory methods. One of the first works in this area is that by I. Prigogine Prigogine \& Xhrouet (1949), followed by studies Present (1960), and Ludwig \& Heil (1960). The effect of non-equilibrium distributions on the chemical reaction rate coefficients was considered in Shizgal \& Karplus (1970). Later on, this effect was studied using various distributions of reacting gas molecules over the internal energy (see, for instance, Refs. Belouaggadia \& Brun (1997); Knab (1996))). Most of these models are based on thermally-equilibrium distributions or non-equilibrium Boltzmann distributions over the vibrational energy of the reagents. More rigorous models of non-equilibrium kinetics in a flow take into account the non-Boltzmann quasi-stationary distributions or the state-to-state vibrational and chemical kinetics Kustova et al. (1999); Nagnibeda \& Kustova (2009). The influence of state-to-state and multi-temperature distributions on reaction rates in particular flows are studied in Kustova \& Nagnibeda (2000); Kustova et al. (2003). Recently the comparison of kinetic models for transport properties in reacting gas flows has been discussed in Kustova \& Nagnibeda (2011).

In the present contribution, we propose mathematical description for the chemical kinetics in gas flows on the basis of the Chapman-Enskog method, generalized for strongly non-equilibrium reacting gas mixtures. 
First, we consider the one-temperature model for the non-equilibrium chemical kinetics in thermally equilibrium gas flows or deviating weakly from thermal equilibrium state. Then, the models for vibrational-chemical coupling in gas flows are derived from the kinetic theory taking into account state-to-state and multi-temperature vibrational distributions.

The influence of non-equilibrium distributions, gas compressibility and space inhomogeneity on the reaction rates for different processes is discussed.

\section{One-temperature model for non-equilibrium kinetics}

\subsection{Kinetic equations. Distribution functions}

We consider strong non-equilibrium chemical kinetics in a flow under the following conditions for relaxation times

$$
\tau_{\text {el }}<\tau_{\text {int }} \ll \tau_{\text {react }} \sim \theta
$$

Here $\tau_{\text {el }}, \tau_{\text {int }}, \tau_{\text {react }}$ and $\theta$ are mean times for relaxation of translation and internal degrees of freedom, chemical reactions and gas dynamic parameters changing respectively. The kinetic equations for the distribution functions have the form Nagnibeda \& Kustova (2009):

$$
\frac{\partial f_{c i j}}{\partial t}+\mathbf{u}_{c} \cdot \nabla f_{c i j}=\frac{1}{\varepsilon} J_{c i j}^{r a p}+J_{c i j}^{s l}
$$

$\varepsilon=\tau_{\text {rap }} / \tau_{s l} \sim \tau_{\text {rap }} / \theta \ll 1$ is the small parameter, $J_{c i j}^{\text {rap }}$ and $J_{c i j}^{s l}$ are the collision integral operators for rapid and slow processes, $c, i, j$ denote chemical species, vibrational and rotational levels respectively, $\mathbf{r}, \mathbf{u}, t$ are coordinates, molecular velocities and time. Under condition (2), integral operators of rapid processes describe elastic collisions and collisions with rotational and vibrational energies change and can be written in the form

$$
J_{c i j}^{r a p}=J_{c i j}^{e l}+J_{c i j}^{r o t}+J_{c i j}^{v i b r}=J_{c i j}^{e l}+J_{c i j}^{i n t}
$$

The operator of slow processes $J_{c i j}^{s l}=\int_{c i j}^{r e a c t}$ includes the integrals of reactive collisions and describes exchange reactions

$$
A_{c}\left(\mathbf{u}_{c}, i, j\right)+A_{d}\left(\mathbf{u}_{d}, k, l\right) \rightleftharpoons A_{c^{\prime}}\left(\mathbf{u}_{c^{\prime}}, i^{\prime}, j^{\prime}\right)+A_{d^{\prime}}\left(\mathbf{u}_{d^{\prime}}, k^{\prime}, l^{\prime}\right),
$$

and dissociation-recombination reactions

$$
A_{c}\left(\mathbf{u}_{c}, i, j\right)+A_{d}\left(\mathbf{u}_{d}, k, l\right) \rightleftharpoons A_{c^{\prime}}\left(\mathbf{u}_{c^{\prime}}\right)+A_{f^{\prime}}\left(\mathbf{u}_{f^{\prime}}\right)+A_{d}\left(\mathbf{u}_{d^{\prime}}^{\prime}, k, l\right),
$$

$c^{\prime}, f^{\prime}$ are the atomic species forming as reaction products; $\mathbf{u}_{c^{\prime}}, \mathbf{u}_{f^{\prime}}, \mathbf{u}_{d}^{\prime}$ are the particle velocities after the collision. For the simplicity we consider dissociation of only diatomic molecules, therefore products of dissociation are only atoms. In addition to this, it is commonly supposed that the dissociation cross section does not depend on the internal state of a partner in the reaction, and this state does not vary as a result of dissociation and recombination.

The collision operator $J_{c i j}^{r e a c t}$ represents the sum of two terms, $J_{c i j}^{e x}$ and $J_{c i j}^{d i s s}$. Expressions for these operators are given, for instance, in Alexeev et al. (1994); Ern \& Giovangigli (1998); 
Kuščer (1991); Ludwig \& Heil (1960); Nagnibeda \& Kustova (2009); Rydalevskaya (1977):

$$
\begin{aligned}
& J_{c i j}^{e x}=\sum_{d c^{\prime} d^{\prime}} \sum_{k i^{\prime} k^{\prime}} \sum_{l j^{\prime} l^{\prime}} \int\left[f_{c^{\prime} i^{\prime} j^{\prime}} f_{d^{\prime} k^{\prime} l^{\prime}} \frac{s_{i j}^{c} s_{k l}^{d}}{s_{i^{\prime} j^{\prime}}^{c^{\prime}} d_{k^{\prime} l^{\prime}}^{d^{\prime}}}\left(\frac{m_{c} m_{d}}{m_{c^{\prime}} m_{d^{\prime}}}\right)^{3}-f_{c i j} f_{d k l}\right] g \sigma_{c d, i j k l}^{c^{\prime} d^{\prime}, i^{\prime} j^{\prime} k^{\prime} l^{\prime}} d^{2} \Omega d \mathbf{u}_{d,} \\
& J_{c i j}^{d i s s}=\sum_{d} \sum_{k} \sum_{l} \int\left[f_{d k l}^{\prime} f_{c^{\prime}} f_{f^{\prime}} h^{3} s_{i j}^{c}\left(\frac{m_{c}}{m_{c^{\prime}} m_{f^{\prime}}}\right)^{3}-f_{c i j} f_{d k l}\right] g \sigma_{c i j, d}^{d i s s} d \mathbf{u}_{d} d \mathbf{u}_{c^{\prime}} d \mathbf{u}_{f^{\prime}} d \mathbf{u}_{d}^{\prime} .
\end{aligned}
$$

In Eq. (6), $\sigma_{c d, i j k l}^{c^{\prime} d^{\prime}, i^{\prime} j^{\prime} k^{\prime} l^{\prime}}$ is the differential cross section of the exchange reaction, and the distribution functions after the collision are denoted $f_{c^{\prime} i^{\prime} j^{\prime}}=f_{c^{\prime} i^{\prime} j^{\prime}}\left(\mathbf{r}, \mathbf{u}_{c^{\prime}}, t\right), f_{d^{\prime} k^{\prime} l^{\prime}}=$ $f_{d^{\prime} k^{\prime} l^{\prime}}\left(\mathbf{r}, \mathbf{u}_{d^{\prime}}, t\right)$; in Eq. (7), $\sigma_{c i j, d}^{\text {diss }}\left(\mathbf{u}_{c}, \mathbf{u}_{d}, \mathbf{u}_{c^{\prime}}, \mathbf{u}_{f^{\prime}}, \mathbf{u}_{d}^{\prime}\right)$ is the formal cross section of dissociation, $f_{c^{\prime}}=f_{c^{\prime}}\left(\mathbf{r}, \mathbf{u}_{c^{\prime}}, t\right), f_{f^{\prime}}=f_{f^{\prime}}\left(\mathbf{r}, \mathbf{u}_{f^{\prime}}, t\right)$ are the distribution functions of atomic dissociation products; $f_{d k l}^{\prime}=f_{d k l}\left(\mathbf{r}, \mathbf{u}_{d}^{\prime}, t\right), h$ is the Plank constant, $m_{c}$ is the mass of a molecule $c, s_{i j}^{c}$ is the statistical weight of the internal states $i$ and $j$ of a component $c, g$ is the relative velocity, $\Omega$ is the solid angle in which a molecule appear after a collision.

Expressions (6), (7) are written taking into account the principle of microscopic reversibility for reactive collisions considered in Alexeev et al. (1994); Ern \& Giovangigli (1998); Kuščer (1991); Ludwig \& Heil (1960); Rydalevskaya (1977):

$$
\begin{aligned}
& s_{i^{\prime} j^{\prime}}^{c^{\prime}} d_{k^{\prime} l^{\prime}}^{d^{\prime}} m_{c^{\prime}}^{2} m_{d^{\prime}}^{2} g^{\prime 2} \sigma_{c^{\prime} d^{\prime}, i^{\prime} j^{\prime} k^{\prime} l^{\prime}}^{c d, i j{ }^{\prime}}\left(\mathbf{g}^{\prime}, \Omega\right)=s_{i j}^{c} s_{k l}^{d} m_{c}^{2} m_{d}^{2} g^{2} \sigma_{c d, i j k l}^{c^{\prime} d^{\prime}, i^{\prime} j^{\prime} k^{\prime} l^{\prime}}\left(\mathbf{g}, \Omega^{\prime}\right), \\
& \frac{m_{c^{\prime}}^{3} m_{f^{\prime}}^{3}}{h^{3}} \sigma_{c^{\prime} f^{\prime} d}^{r e c, c i j}\left(\mathbf{u}_{c^{\prime}}, \mathbf{u}_{f^{\prime}}, \mathbf{u}_{d}^{\prime}, \mathbf{u}_{c}, \mathbf{u}_{d}\right)=s_{i j}^{c} m_{c}^{3} g \sigma_{c i j, d}^{d i s s}\left(\mathbf{u}_{c}, \mathbf{u}_{d}, \mathbf{u}_{c^{\prime}}, \mathbf{u}_{f^{\prime}}, \mathbf{u}_{d}^{\prime}\right),
\end{aligned}
$$

$\sigma_{c^{\prime} f^{\prime} d}^{r e c, c i j}$ is the probability density for a triple collision resulting in dissociation.

In the frame of the method proposed in Kustova \& Nagnibeda (1998); Nagnibeda \& Kustova (2009) for the solution of Eqs. (2), the distribution functions are expanded in a power series of the small parameter $\varepsilon$. The peculiarity of the modified Chapman-Enskog method is that the distribution functions and macroscopic parameters are determined by the collision invariants of the most frequent collisions. Under condition (1), the set of collision invariants contains the invariants of any collision (momentum and total energy) and the additional invariants of rapid processes. In our case, these additional invariants are any variables independent of the velocity and internal energy and depending arbitrary on chemical species $c$ because chemical reactions are supposed to be frozen in rapid processes This set of collision invariants provides the following set of macroscopic parameters for a closed flow description: number densities of species $n_{c}(\mathbf{r}, t)(c=1, \ldots, L)$, gas velocity $\mathbf{v}(\mathbf{r}, t)$ and temperature $T(\mathbf{r}, t)$.

\subsection{Governing equations. Reaction rates}

Closed set of equations of the flow are derived from the kinetic equations (2). Integrating these equations over velocities and summing over the internal energy levels we obtain equations of chemical kinetics in the flow. Multiplying kinetic equations by the collision invariants of any collision, integrating over the velocity and summing over the internal energy levels, we obtain the conservation equations for the momentum and total energy. Finally the set of governing equations for macroscopic parameters $n_{\mathcal{c}}(\mathbf{r}, t), \mathbf{v}(\mathbf{r}, t), T(\mathbf{r}, t)$ takes the form: 


$$
\begin{aligned}
& \frac{d n_{c}}{d t}+n_{c} \nabla \cdot \mathbf{v}+\nabla \cdot\left(n_{c} \mathbf{V}_{c}\right)=R_{c}^{\text {react }}, c=1, . ., L, \\
& \rho \frac{d \mathbf{v}}{d t}+\nabla \cdot \boldsymbol{P}=0, \\
& \rho \frac{d U}{d t}+\nabla \cdot \mathbf{q}+\boldsymbol{P}: \nabla \mathbf{v}=0 .
\end{aligned}
$$

Here $U$ is the total energy per unit mass which is the function of temperature and species number densities, $\boldsymbol{P}$ is the pressure tensor, $\mathbf{q}$ is the total heat flux, $\mathbf{V}_{i}$ are the diffusion velocities.

The production terms in Eqs. (10) take the form

$$
\begin{aligned}
R_{c}^{e x} & =\sum_{d c^{\prime} d^{\prime}}\left(n_{\mathcal{c}^{\prime}} n_{d^{\prime}} k_{c^{\prime} c}^{d^{\prime} d}-n_{c} n_{d} k_{c c^{\prime}}^{d d^{\prime}}\right), \\
R_{c}^{d i s s} & =\sum_{d} n_{d}\left(n_{c^{\prime}} n_{f^{\prime}} k_{r e c, c}^{d}-n_{c} k_{c, d i s s}^{d}\right) .
\end{aligned}
$$

Production terms (13) (14) contain the rate coefficients of exchange chemical reactions $k_{c c^{\prime}}^{d d^{\prime}}$, dissociation $k_{c, \text { diss }}^{d}$, and recombination $k_{r e c, c}^{d}$ Nagnibeda \& Kustova (2009).

For practical calculations it is more suitable to use component mass fractions $\alpha_{c}=\rho_{c} / \rho$ instead of number densities $n_{c}$ ( $\rho$ is the total mass density, $\rho_{c}$ is the component $c$ mass density). In this case, the set of macroscopic parameters includes $\alpha_{c}(\mathbf{r}, t)(c=1, \ldots, L), \mathbf{v}(\mathbf{r}, t), T(\mathbf{r}, t)$. The equations of chemical kinetics take the form:

$$
\rho \frac{d \alpha_{c}}{d t}=-\nabla \cdot\left(\rho_{c} \mathbf{V}_{c}\right)+\sum_{r} \dot{\xi}_{r} v_{r c} M_{c}, \quad c=1, . ., L
$$

here $M_{c}$ is the component molar mass, $\dot{\xi}_{r}$ is the chemical reaction rate for reaction $r(r=$ $1, \ldots, R, R$ is the number of reactions in a mixture), $v_{r c}=v_{r c}^{(p)}-v_{r c}^{(r)}$ is global stoichiometric coefficient, $v_{r c}^{(r)}, v_{r c}^{(p)}$ are the stoichiometric coefficients of reactants and products.

The source terms are defined by expressions:

$$
\sum_{r} \dot{\xi}_{r} v_{r c} M_{c}=m_{c} \sum_{i j} \int J_{c i j}^{s l} d \mathbf{u}_{c}=m_{c} \sum_{i j} \int\left(J_{c i j}^{e x}+J_{c i j}^{d i s s}\right) d \mathbf{u}_{c} .
$$

Let us introduce the rate coefficients of forward and backward reactions $k_{f, r}, k_{b, r}$. For exchange reaction (4) $(r=e x)$, and recombination-dissociation reaction (5) $(r=$ diss) they have the form:

$$
\begin{gathered}
v_{e x, c} k_{f, e x}=-\mathcal{N}_{A} \sum_{j j^{\prime} l^{\prime} l_{i}} \sum_{i i^{\prime} k^{\prime}} \int \frac{f_{c i j} f_{d k l}}{n_{c} n_{d}} g \sigma_{c d, i j k l}^{c^{\prime} d^{\prime}, i^{\prime} j^{\prime} k^{\prime} k^{\prime} l^{\prime}} d^{2} \Omega d \mathbf{u}_{d} d \mathbf{u}_{c}, \\
v_{e x, c} k_{b, e x}=-\mathcal{N}_{A} \sum_{j l j^{\prime} l^{\prime}} \sum_{i k i^{\prime} k^{\prime}} \int \frac{f_{c^{\prime} i^{\prime} j^{\prime}} f_{d^{\prime} k^{\prime} l^{\prime}}}{n_{c^{\prime}} n_{d^{\prime}}} g^{\prime} \sigma_{c^{\prime} d^{\prime}, i^{\prime} j^{\prime} k^{\prime} l^{\prime}}^{c d, i j k l} d^{2} \Omega d \mathbf{u}_{d^{\prime}} d \mathbf{u}_{c^{\prime}},
\end{gathered}
$$




$$
\begin{gathered}
v_{d i s s, c} k_{f, d i s s}=-\mathcal{N}_{A} \sum_{j l} \sum_{i k} \int \frac{f_{c i j} f_{d k l}}{n_{c} n_{d}} g \sigma_{c i j, d}^{d i s s} d \mathbf{u}_{c} d \mathbf{u}_{d} d \mathbf{u}_{c^{\prime}} d \mathbf{u}_{f^{\prime}} d \mathbf{u}_{d \prime}^{\prime}, \\
v_{d i s s, c} k_{b, d i s s}=-\mathcal{N}_{A}^{2} \sum_{j l} \sum_{i k} \int \frac{f_{c^{\prime}} f_{f^{\prime}} f_{d k l}^{\prime}}{n_{c^{\prime}} n_{f^{\prime}} n_{d}} \sigma_{c^{\prime} f^{\prime} d}^{r e c, c i j} d \mathbf{u}_{c^{\prime}} d \mathbf{u}_{f^{\prime}} d \mathbf{u}_{d}^{\prime} d \mathbf{u}_{c} d \mathbf{u}_{d} .
\end{gathered}
$$

Rate coefficients (17)-(20) are connected with those appearing in equations (13), (14) by the relations:

$$
k_{f, r}=-\frac{\mathcal{N}_{A}}{v_{r c}} k_{c c^{\prime}}^{d d^{\prime}}, \quad k_{b, r}=-\frac{\mathcal{N}_{A}}{v_{r c}} k_{c^{\prime} c}^{d^{\prime} d}
$$

for exchange reactions, and

$$
k_{f, r}=-\frac{\mathcal{N}_{A}}{v_{r c}} k_{c, \text { diss }}^{d}, \quad k_{b, r}=-\frac{\mathcal{N}_{A}^{2}}{v_{r c}} k_{r e c, c}^{d}
$$

for dissociation and recombination reactions, $\mathcal{N}_{A}$ is the Avogadro number.

Using equations (16), (17)-(20), we can write the expression for the reaction rate $\dot{\xi}_{r}$ in the conventional form:

$$
\dot{\xi}_{r}=k_{f, r} \prod_{c=1}^{L}\left(\frac{\rho_{c}}{M_{c}}\right)^{v_{r c}^{(r)}}-k_{b, r} \prod_{c=1}^{L}\left(\frac{\rho_{c}}{M_{c}}\right)^{v_{r c}^{(p)}} .
$$

One can notice that the general expressions for the rate coefficients depend on the cross-section of corresponding reactions as well as on the distribution functions, and, consequently, on the approximation of the Chapman-Enskog method.

\subsection{Zero-order reaction-rate coefficients}

The zero-order solution of Eqs. (2) for molecular species has the form of the Maxwell-Boltzmann distributions

$$
f_{c i j}^{(0)}=\left(\frac{m_{c}}{2 \pi k T}\right)^{3 / 2} \frac{n_{c}}{Z_{c}^{i n t}(T)} s_{i j}^{c} \exp \left(-\frac{m_{c} c_{c}^{2}}{2 k T}-\frac{\varepsilon_{i j}^{c}}{k T}\right),
$$

with the internal partition function $Z_{c}^{\text {int }}$ given by

$$
Z_{c}^{i n t}(T)=\sum_{i j} s_{i j}^{c} \exp \left(-\frac{\varepsilon_{i j}^{c}}{k T}\right) .
$$

Here $\varepsilon_{i j}^{c}$ is the internal energy of a molecule at the $i$ th vibrational and $j$ th rotational levels, $k$ is the Boltzmann constant, $\mathbf{c}_{c}=\mathbf{u}_{c}-\mathbf{v}$ is the peculiar velocity. The zero-order distribution function for atomic species reads

$$
f_{c}^{(0)}=\left(\frac{m_{c}}{2 \pi k T}\right)^{3 / 2} n_{c} \exp \left(-\frac{m_{c} c_{c}^{2}}{2 k T}\right) .
$$

The zero order transport terms in the flow equations take the form $\mathbf{V}_{c}^{(0)}=0, \boldsymbol{P}^{(0)}=p \boldsymbol{I}$, $\mathbf{q}^{(0)}=0$, the pressure is $p=n k T, n$ is the total number density. Thus, in the zero-order 
(Euler) approximation, the governing equations describe non-equilibrium chemical kinetics in a thermally equilibrium inviscid non-conducting gas mixture:

$$
\begin{aligned}
& \rho \frac{d \alpha_{c}}{d t}=\sum_{r} \dot{\xi}_{r}^{(0)} v_{r c} M_{\mathcal{c}}, \quad c=1, . ., L, \\
& \rho \frac{d \mathbf{v}}{d t}=-\nabla p, \\
& \rho \frac{d U}{d t}=-p \nabla \cdot \mathbf{v} .
\end{aligned}
$$

The right-hand side in Eqs. (26) contain the zero-order reaction rates:

$$
\dot{\xi}_{r}^{(0)}=k_{f, r}^{(0)} \prod_{c=1}^{L}\left(\frac{\rho_{c}}{M_{c}}\right)^{v_{r c}^{(r)}}-k_{b, r}^{(0)} \prod_{c=1}^{L}\left(\frac{\rho_{c}}{M_{c}}\right)^{v_{r c}^{(p)}} .
$$

Here $k_{f, r}^{(0)}, k_{b, r}^{(0)}$ are the thermal-equilibrium reaction-rate coefficients. For the exchange reaction (4), when the partner $A_{d}$ is a molecule, $k_{f, e x}^{(0)}, k_{b, e x}^{(0)}$ are given by:

$$
\begin{gathered}
v_{e x, c} k_{f, e x}^{(0)}(T)=-\frac{\mathcal{N}_{A}}{Z_{c}^{\text {int }}(T) Z_{d}^{\text {int }}(T)}\left(\frac{m_{c d}}{2 \pi k T}\right)^{3 / 2} \sum_{i k i^{\prime} k^{\prime}} \sum_{j l j^{\prime} l^{\prime}} \int \exp \left(-\frac{m_{c d} g^{2}}{2 k T}\right) \times \\
s_{i j}^{c} s_{k l}^{d} \exp \left(-\frac{\varepsilon_{i j}^{c}+\varepsilon_{k l}^{d}}{k T}\right) g^{3} \sigma_{c d, i j k l}^{c^{\prime} d^{\prime}, i^{\prime} j^{\prime} k^{\prime} l^{\prime}}(g, \Omega) d g d^{2} \Omega, \\
v_{e x, c} k_{b, e x}^{(0)}(T)=-\frac{\mathcal{N}_{A}}{Z_{c^{\prime}}^{\text {int }}(T) Z_{d^{\prime}}^{\text {int }}(T)}\left(\frac{m_{c^{\prime} d^{\prime}}}{2 \pi k T}\right)^{3 / 2} \sum_{i k i^{\prime} k^{\prime} j l j^{\prime} l^{\prime}} \sum_{j} \exp \left(-\frac{m_{c^{\prime} d^{\prime}} g^{2}}{2 k T}\right) \times \\
s_{i^{\prime} j^{\prime}}^{c^{\prime}} k_{k^{\prime} l^{\prime}}^{d^{\prime}} \exp \left(-\frac{\varepsilon_{i^{\prime} j^{\prime}}^{c^{\prime}}+\varepsilon_{k^{\prime} l^{\prime}}^{d^{\prime}}}{k T}\right) g^{\prime 3} \sigma_{c^{\prime} d^{\prime}, i^{\prime} j^{\prime} k^{\prime} l^{\prime}}^{c d, i j k l}\left(g^{\prime}, \Omega\right) d g^{\prime} d^{2} \Omega,
\end{gathered}
$$

and if $A_{d}$ is an atom,

$$
\begin{gathered}
v_{e x, c} k_{f, e x}^{(0)}(T)=-\frac{\mathcal{N}_{A}}{Z_{c}^{i n t}(T)}\left(\frac{m_{c d}}{2 \pi k T}\right)^{3 / 2} \sum_{i i^{\prime}} \sum_{j j^{\prime}} \int \exp \left(-\frac{m_{c d} g^{2}}{2 k T}\right) \times \\
s_{i j}^{c} \exp \left(-\frac{\varepsilon_{i j}^{c}}{k T}\right) g^{3} \sigma_{c d, i j}^{c^{\prime} d^{\prime}, i^{\prime} j^{\prime}}(g, \Omega) d g d^{2} \Omega . \\
v_{e x, c} k_{b, e x}^{(0)}(T)=-\frac{\mathcal{N}_{A}}{Z_{c^{\prime}}^{i n t}(T)}\left(\frac{m_{c^{\prime} d^{\prime}}}{2 \pi k T}\right)^{3 / 2} \sum_{i i^{\prime}} \sum_{j j^{\prime}} \int \exp \left(-\frac{m_{c^{\prime} d^{\prime}} g^{\prime 2}}{2 k T}\right) \times \\
s_{i^{\prime} j^{\prime}}^{c^{\prime}} \exp \left(-\frac{\varepsilon_{i^{\prime} j^{\prime}}^{c^{\prime}}}{k T}\right) g^{\prime 3} \sigma_{c^{\prime} d^{\prime}, i^{\prime} j^{\prime}}^{c d, i j}\left(g^{\prime}, \Omega\right) d g^{\prime} d^{2} \Omega .
\end{gathered}
$$


For the dissociation reaction (5), the zero-order forward-rate coefficient is obtained in the form

$$
\begin{gathered}
v_{\text {diss }, c} k_{f, d i s s}^{(0)}(T)=-\frac{4 \pi \mathcal{N}_{A}}{Z_{c}^{i n t}(T)}\left(\frac{m_{c d}}{2 \pi k T}\right)^{3 / 2} \sum_{i j} \int \exp \left(-\frac{m_{c d} g^{2}}{2 k T}\right) \times \\
s_{i j}^{c} \exp \left(-\frac{\varepsilon_{i j}^{c}}{k T}\right) g^{3} \sigma_{c i j, d}^{d i s s}\left(g, \mathbf{u}_{c^{\prime}}, \mathbf{u}_{f^{\prime}}, \mathbf{u}_{d}^{\prime}\right) d g d \mathbf{u}_{c^{\prime}} d \mathbf{u}_{f^{\prime}} d \mathbf{u}_{d}^{\prime} .
\end{gathered}
$$

The zero-order recombination (backward for dissociation) rate coefficient reads

$$
\begin{aligned}
v_{\text {diss }, c} k_{b, \text { diss }}^{(0)}(T)= & -\mathcal{N}_{A}^{2} \frac{\left(m_{c^{\prime}} m_{f^{\prime}} m_{d}\right)^{3 / 2}}{(2 \pi k T)^{9 / 2}} \sum_{i j} \int \exp \left(-\frac{m_{c^{\prime}} c_{c^{\prime}}^{2}}{2 k T}-\frac{m_{f^{\prime}} c_{f^{\prime}}^{2}}{2 k T}-\frac{m_{d} c_{d}^{2}}{2 k T}\right) \times \\
& \sigma_{c^{\prime} f^{\prime} d}^{r e c, c i j}\left(\mathbf{u}_{c}, \mathbf{u}_{d}, \mathbf{u}_{c^{\prime}}, \mathbf{u}_{f^{\prime}}, \mathbf{u}_{d}^{\prime}\right) d \mathbf{u}_{c} d \mathbf{u}_{d} d \mathbf{u}_{c^{\prime}} d \mathbf{u}_{f^{\prime}} d \mathbf{u}_{d}^{\prime} .
\end{aligned}
$$

Thus, if the cross-sections of the corresponding reactions are known, the zero-order rate coefficients can easily be calculated. However, for practical applications, phenomenological models such as the Arrhenius one are commonly used.

Using the detailed balance principle (8)-(9), one can obtain the ratios of forward and backward reaction rate coefficients:

$$
\begin{gathered}
K_{e x}^{(0)}(T)=\frac{k_{b, e x}^{(0)}(T)}{k_{f, e x}^{(0)}(T)}=\left(\frac{m_{c} m_{d}}{m_{c^{\prime}} m_{d^{\prime}}}\right)^{3 / 2} \frac{Z_{c}^{\text {int }}(T) Z_{d}^{\text {int }}(T)}{Z_{c^{\prime}}^{\text {int }}(T) Z_{d^{\prime}}^{\text {int }}(T)} \exp \left(\frac{D_{c}+D_{d}-D_{c^{\prime}}-D_{d^{\prime}}}{k T}\right), \\
K_{\text {diss }}^{(0)}(T)=\frac{k_{b, \text { diss }}^{(0)}}{k_{f, \text { diss }}^{(0)}}=\mathcal{N}_{A}\left(\frac{m_{c}}{m_{c^{\prime}} m_{f^{\prime}}}\right)^{3 / 2} h^{3}(2 \pi k T)^{-3 / 2} Z_{c}^{\text {int }}(T) \exp \left(\frac{D_{c}}{k T}\right),
\end{gathered}
$$

$D_{c}$ is the dissociation energy of molecule $c, D_{c}+D_{d}-D_{c^{\prime}}-D_{d^{\prime}}$ is heat effect of an exchange reaction. Formulas (36), (37) express the chemical-equilibrium constants well known from thermodynamics and hold only for Maxwell-Boltzmann distributions over velocity and internal energy.

\subsection{First order reaction rate coefficients. Chemical kinetics in viscous gases}

First-order distribution functions are obtained in Nagnibeda \& Kustova (2009) in the form

$$
f_{c i j}^{(1)}=f_{c i j}^{(0)}\left(-\frac{1}{n} \mathbf{A}_{c i j} \cdot \nabla \ln T-\frac{1}{n} \sum_{d} \mathbf{D}_{c i j}^{d} \cdot \mathbf{d}_{d}-\frac{1}{n} \mathbf{B}_{c i j}: \nabla \mathbf{v}-\frac{1}{n} F_{c i j} \nabla \cdot \mathbf{v}-\frac{1}{n} G_{c i j}\right) .
$$

Functions $\mathbf{A}_{c i j}, \mathbf{B}_{c i j}, \mathbf{D}_{c i j}^{d}, F_{c i j}$ and $G_{c i j}$ satisfy the linear integral equations with the linearized operators of elastic collisions and inelastic ones with internal energy transitions.

Let us consider the first-order transport terms in equations Eqs. (10)-(12). For the viscous stress tensor we obtain

$$
\boldsymbol{P}=\left(p-p_{\text {rel }}\right) \boldsymbol{I}-2 \eta \boldsymbol{S}-\zeta \nabla \cdot \mathbf{v} \boldsymbol{I} .
$$

Here, $p_{\text {rel }}$ is the relaxation pressure, $\eta$ and $\zeta$ are the coefficients of shear and bulk viscosity. In the one-temperature approach, the additional terms connected to the bulk viscosity and 
relaxation pressure appear in the diagonal terms of the stress tensor due to rapid inelastic internal energy transitions. The existence of the relaxation pressure is caused also by slow processes of chemical reactions proceeding on the gas-dynamic time scale.

The transport coefficients in the expression (39) can be written in terms of functions $\mathbf{B}_{c i j}, F_{c i j}$, and $G_{c i j}$ :

$$
\eta=\frac{k T}{10}[\mathbf{B}, \mathbf{B}], \quad \zeta=k T[F, F], \quad p_{r e l}=k T[F, G] .
$$

In these formulae, $[A, B]$ (where $A, B$ are arbitrary functions of molecular velocities) denotes a bilinear form depending on the linearized integral collision operator for rapid processes. In the kinetic theory, such bilinear forms are basically called bracket integrals. The bracket integrals in the expressions (40) are introduced in Nagnibeda \& Kustova (2009) similarly to those defined in Ferziger \& Kaper (1972) for a non-reacting gas mixture under the conditions of weak deviations from the equilibrium.

The diffusion velocity and the total energy flux in the considered approach are specified by the functions $\mathbf{D}_{c i j}^{d}, \mathbf{A}_{c i j}^{d}$ also depending on the cross sections of rapid processes and are studied in Kustova \& Nagnibeda (2011).

Thus, the governing equations (10)-(12) with the first order transport terms describe a flow of reacting mixture of viscous gases with strong non-equilibrium chemical reactions in the Navier-Stokes approximation. Transport properties in the one-temperature approach in reacting gas mixtures are considered in Ern \& Giovangigli (1994); Kustova (2009); Kustova et al. (2008); Nagnibeda \& Kustova (2009).

The chemical reaction rate coefficients contributing to the production terms $R_{c}^{\text {react }}$ in the equations (10) or to the reaction rates in the equations (15) in the first-order approximation are defined by the first order distribution functions (38) and depend on the cross sections of reactive collisions.

The chemical-reaction rate in Eqs. (15) in the first-order approximation has the form (23) where

$$
\begin{aligned}
& k_{f, r}=k_{f, r}^{(0)}(T)-\bar{k}_{f, r}^{(1)}\left(\alpha_{1}, \ldots, \alpha_{L}, \rho, T\right)-\tilde{k}_{f, r}^{(1)}\left(\alpha_{1}, \ldots, \alpha_{L}, \rho, T\right), \\
& k_{b, r}=k_{b, r}^{(0)}(T)-\bar{k}_{b, r}^{(1)}\left(\alpha_{1}, \ldots, \alpha_{L}, \rho, T\right)-\tilde{k}_{b, r}^{(1)}\left(\alpha_{1}, \ldots, \alpha_{L}, \rho, T\right) .
\end{aligned}
$$

Quantities $\bar{k}_{f, r}^{(1)}, \tilde{k}_{f, r}^{(1)}, \bar{k}_{b, r}^{(1)}, \tilde{k}_{b, r}^{(1)}$ express first-order corrections to the reaction-rate coefficients (17)-(20). The terms $\bar{k}_{f, r}^{(1)}, \bar{k}_{b, r}^{(1)}$ are due to deviations from Maxwell-Boltzmann distributions over velocities and internal energies whereas the terms $\tilde{k}_{f, r}^{(1)}, \tilde{k}_{b, r}^{(1)}$ are due to spatial non-homogeneity. If internal degrees of freedom are neglected, the coefficients $\tilde{k}_{f, r}^{(1)}$ and $\tilde{k}_{b, r}^{(1)}$ vanish.

The first-order corrections to the reaction-rate coefficients are defined by the expressions:

$$
v_{e x, c} \bar{k}_{f, e x}^{(1)}=-\frac{\mathcal{N}_{A}}{n} \sum_{i k i^{\prime} k^{\prime}} \sum_{j j^{\prime} l^{\prime}} \int \frac{f_{c i j}^{(0)} f_{d k l}^{(0)}}{n_{c} n_{d}}\left(G_{c i j}+G_{d k l}\right) g \sigma_{c d, i j k l}^{c^{\prime} d^{\prime}, i^{\prime} j^{\prime} k^{\prime} l^{\prime}} d^{2} \Omega d \mathbf{u}_{d} d \mathbf{u}_{c,}
$$




$$
\begin{aligned}
& v_{e x, c} \tilde{k}_{f, e x}^{(1)}=-\nabla \cdot \mathbf{v} \frac{\mathcal{N}_{A}}{n} \sum_{i k i^{\prime} k^{\prime}} \sum_{j j^{\prime} l^{\prime}} \int \frac{f_{c i j}^{(0)} f_{d k l}^{(0)}}{n_{c} n_{d}}\left(F_{c i j}+F_{d k l}\right) g \sigma_{c d, i j k l}^{c^{\prime} d^{\prime}, i^{\prime} j^{\prime} k^{\prime} l^{\prime}} d^{2} \Omega d \mathbf{u}_{d} d \mathbf{u}_{c}, \\
& v_{e x, c} \bar{k}_{b, e x}^{(1)}=-\frac{\mathcal{N}_{A}}{n} \sum_{i k i^{\prime} k^{\prime}} \sum_{j l^{\prime} l^{\prime}} \int \frac{f_{\mathcal{c}^{\prime} i^{\prime} j^{\prime}}^{(0)} f_{d^{\prime} k^{\prime} l^{\prime}}^{(0)}}{n_{c^{\prime}} n_{d^{\prime}}}\left(G_{c^{\prime} i^{\prime} j^{\prime}}+G_{d^{\prime} k^{\prime} l^{\prime}}\right) g^{\prime} \sigma_{c^{\prime} d^{\prime}, i^{\prime} j^{\prime} k^{\prime} l^{\prime}}^{c d, i j l} d^{2} \Omega d \mathbf{u}_{d^{\prime}} d \mathbf{u}_{c^{\prime}},
\end{aligned}
$$

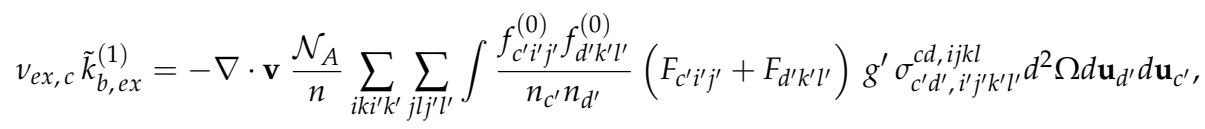

$$
\begin{aligned}
& v_{d i s s, c} \bar{k}_{f, d i s s}^{(1)}=-\frac{\mathcal{N}_{A}}{n} \sum_{i k i^{\prime} k^{\prime}} \sum_{j l j^{\prime} l^{\prime}} \int \frac{f_{c i j}^{(0)} f_{d k l}^{(0)}}{n_{c} n_{d}}\left(G_{c i j}+G_{d k l}\right) g \sigma_{c i j, d}^{d i s s} d \mathbf{u}_{c} d \mathbf{u}_{d} d \mathbf{u}_{c^{\prime}} d \mathbf{u}_{f^{\prime}} d \mathbf{u}_{d}^{\prime}, \\
& v_{d i s s, c} \tilde{k}_{f, d i s s}^{(1)}=-\nabla \cdot \mathbf{v} \frac{\mathcal{N}_{A}}{n} \sum_{i k i^{\prime} k^{\prime}} \sum_{j l j^{\prime} l^{\prime}} \int \frac{f_{c i j}^{(0)} f_{d k l}^{(0)}}{n_{c} n_{d}}\left(F_{c i j}+F_{d k l}\right) g \sigma_{c i j, d}^{d i s s} d \mathbf{u}_{c} d \mathbf{u}_{d} d \mathbf{u}_{c^{\prime}} d \mathbf{u}_{f^{\prime}} d \mathbf{u}_{d^{\prime}}^{\prime}, \\
& v_{d i s s, c} \bar{k}_{b, \text { diss }}^{(1)}=-\frac{\mathcal{N}_{A}^{2}}{n} \sum_{i k} \sum_{j l} \int \frac{f_{c^{\prime}}^{(0)} f_{f^{\prime}}^{(0)} f_{d k l}^{\prime}(0)}{n_{c^{\prime}} n_{f^{\prime}} n_{d}}\left(G_{c^{\prime}}+G_{f^{\prime}}+G_{d k l}\right) \sigma_{c^{\prime} f^{\prime} d}^{r e c, c i j} d \mathbf{u}_{c} d \mathbf{u}_{d} d \mathbf{u}_{c^{\prime}} d \mathbf{u}_{f^{\prime}} d \mathbf{u}_{d^{\prime}}^{\prime} \text {, } \\
& v_{d i s s, c} \tilde{k}_{b, d i s s}^{(1)}=-\nabla \cdot \mathbf{v} \frac{\mathcal{N}_{A}^{2}}{n} \sum_{i k} \sum_{j l} \int \frac{f_{c^{\prime}}^{(0)} f_{f^{\prime}}^{(0)} f_{d k l}^{\prime(0)}}{n_{c^{\prime}} n_{f^{\prime}} n_{d}}\left(F_{c^{\prime}}+F_{f^{\prime}}+F_{d k l}\right) \sigma_{c^{\prime} f^{\prime} d}^{r e c, c i j} d \mathbf{u}_{c} d \mathbf{u}_{d} d \mathbf{u}_{c^{\prime}} d \mathbf{u}_{f^{\prime}} d \mathbf{u}_{d}^{\prime} .
\end{aligned}
$$

It can be noted that the first-order corrections for the reaction rate coefficients depend on the same functions $F_{c i j}$ and $G_{c i j}$ which define the additional diagonal elements of the pressure tensor connected to the bulk viscosity and relaxation pressure.

Algorithms for the calculation of vector and tensor transport properties and first order corrections to reaction rate coefficients are described in details in Nagnibeda \& Kustova (2009). In Ref. Alexeev \& Grushin (1994), a procedure for the calculation of the first-order reaction rate coefficients has been developed for gases without internal degrees of freedom. In Ref. Kustova et al. (2008), the scalar functions $F_{c i j}$ and $G_{c i j}$ are considered, and transport linear systems for the calculation of bulk viscosity, chemical-reaction contribution to the normal mean stress and first-order reaction rate coefficients are derived taking into account internal energy of molecules. Numerical estimations of the first-order rate coefficients in reacting viscous gas flows remain an open question up to now. In the simulations of viscous flows, the first-order corrections to the rate coefficients are usually neglected as well as relaxation pressure and bulk viscosity. Some results in this field have been recently obtained in Ref. Kustova (2009) where numerical estimations of the normal mean stress and the first order corrections to the dissociation and recombination rates in the mixture $\mathrm{N}_{2} / \mathrm{N}$ have been performed. It is shown that whereas the first-order contribution to the normal mean stress remains small, the first-order corrections to the reaction rates are not negligible in both shock heated and expanding flows. 


\section{State-to-state model for vibrational-chemical coupling}

\subsection{Distribution functions. Governing equations}

In this section, chemical kinetics in multi-component reacting gas mixture flows are studied under the conditions of strong vibrational and chemical non-equilibrium. Experimental results on the relaxation times of various processes demonstrate that, in a wide temperature range, the equilibration of translational and rotational degrees of freedom proceeds much faster compared to the vibrational relaxation and chemical reactions. The characteristic relaxation times satisfy the relation

$$
\tau_{\text {el }}<\tau_{\text {rot }} \ll \tau_{\text {vibr }} \sim \tau_{\text {react }} \sim \theta .
$$

where $\tau_{\text {rot }}, \tau_{v i b r}$ are relaxation times for rotational and vibrational degrees of freedom. Under such conditions, the integral operators in the kinetic equations (2) take the form:

$$
J_{c i j}^{r a p}=J_{c i j}^{e l}+J_{c i j}^{r o t}, \quad J_{c i j}^{s l}=J_{c i j}^{v i b r}+J_{c i j}^{r e a c t} .
$$

In this case, the vibrational-chemical coupling in reacting flows becomes important.

The kinetic equations for the distribution functions in the zero-order Chapman-Enskog approximation have the form:

$$
J_{c i j}^{e l(0)}+J_{c i j}^{r o t(0)}=0 .
$$

In this case, the system of collision invariants for the most frequent collisions includes along with the momentum and a particle total energy, any value independent of the velocity and rotational level $j$ and depending arbitrarily on the vibrational level $i$ and chemical species $c$. This values are conserved at the most frequent collisions because, according to the condition (51), vibrational energy transitions and chemical reactions are forbidden in the rapid processes. Based on the above set of the collision invariants, the solution of Eqs. (53) takes the form

$$
f_{c i j}^{(0)}=\left(\frac{m_{c}}{2 \pi k T}\right)^{3 / 2} s_{j}^{c i} \frac{n_{c i}}{Z_{c i}^{r o t}(T)} \exp \left(-\frac{m_{c} c_{c}^{2}}{2 k T}-\frac{\varepsilon_{j}^{c i}}{k T}\right)
$$

for molecular species, and

$$
f_{c}^{(0)}=\left(\frac{m_{c}}{2 \pi k T}\right)^{3 / 2} n_{c, a} \exp \left(-\frac{m_{c} c_{c}^{2}}{2 k T}\right)
$$

for atomic species. Here $n_{c i}$ is the number density of molecules $c$ at the $i$-th vibrational level, $n_{c, a}$ is the number density of atoms $c, Z_{c i}^{r o t}$ is the partition functions of rotational degrees of freedom:

$$
Z_{c i}^{r o t}(T)=\sum_{j} s_{j}^{c i} \exp \left(-\frac{\varepsilon_{j}^{c i}}{k T}\right),
$$

$\varepsilon_{j}^{c i}$ is the rotational energy of a molecule at the $i$ th vibrational level, $s_{j}^{c i}$ is the rotational statistical weight.

The solution (54) represents the local equilibrium Maxwell-Boltzmann distribution over the velocity and rotational energy levels with the temperature $T$ and strongly non-equilibrium distribution over chemical species and vibrational energy levels. The distribution functions 
(54), (55) are completely specified by the macroscopic gas parameters $n_{c i}(\mathbf{r}, t)(c=1, \ldots, L, i=$ $0,1, \ldots, L c, L_{c}$ is the number of excited vibration levels of molecular species $\left.c\right), T(\mathbf{r}, t)$, and $\mathbf{v}(\mathbf{r}, t)$ and correspond to the set of the collision invariants of rapid processes.

The set of equations for the macroscopic parameters $n_{c i}(\mathbf{r}, t), \mathbf{v}(\mathbf{r}, t)$, and $T(\mathbf{r}, t)$ follows from Eq. (2) with collision operators (52). This system includes equations of state-to-state vibrational and chemical kinetics in a flow Kustova \& Nagnibeda (1998); Nagnibeda \& Kustova (2009):

$$
\frac{d n_{c i}}{d t}+n_{c i} \nabla \cdot \mathbf{v}+\nabla \cdot\left(n_{c i} \mathbf{V}_{c i}\right)=R_{c i}, \quad c=1, \ldots, L, \quad i=0, \ldots, L_{c},
$$

coupled to the conservation equations for the momentum and total energy which formally coincide with Eqs. (11)-(12). Here, $\mathbf{V}_{c i}$ is the diffusion velocity of molecules $\mathrm{c}$ at the vibrational state $i$. The total energy per unit mass $U$ is specified by level populations $n_{c i}(\mathbf{r}, t)$, atomic number densities $n_{\mathcal{c}}(\mathbf{r}, t)$ and gas temperature.

The source terms in equations (57) characterize the variation of the vibrational level populations and atomic number densities caused by different vibrational energy exchanges and chemical reactions and are expressed via the integral operators of slow processes:

$$
R_{c i}=\sum_{j} \int J_{c i j}^{s l} d \mathbf{u}_{c}=R_{c i}^{v i b r}+R_{c i}^{r e a c t} .
$$

The equations (57), (11), (12) provide a detailed description of vibrational and chemical kinetics and flow dynamics for weak deviations from the equilibrium distributions over the velocity and rotational energy levels and arbitrary deviations from the equilibrium for the vibrational degrees of freedom and chemical species. Let us emphasize that for such an approach, the vibrational level populations are included to the set of main macroscopic parameters, and particles of various chemical species in different vibrational states represent the mixture components. The expressions (58) can be written in the form:

$$
\begin{gathered}
R_{c i}^{v i b r}=\sum_{d k i^{\prime} k^{\prime}}\left(n_{c i^{\prime}} n_{d k^{\prime}} k_{c, i^{\prime} i}^{d, k^{\prime} k}-n_{c i} n_{d k} k_{c, i i^{\prime}}^{d, k k^{\prime}}\right), \\
R_{c i}^{r e a c t}=R_{c i}^{e x}+R_{c i}^{d i s s}, \\
R_{c i}^{e x}=\sum_{d c^{\prime} d^{\prime}} \sum_{k^{\prime} k^{\prime}}\left(n_{c^{\prime} i^{\prime}} n_{d^{\prime} k^{\prime}} k_{c^{\prime} i^{\prime}, c i}^{d^{\prime} k^{\prime}, d k}-n_{c i} n_{d k} k_{c i, c^{\prime} i^{\prime}}^{d k, k^{\prime}}\right), \\
R_{c i}^{d i s s}=\sum_{d k} n_{d k}\left(n_{c^{\prime}} n_{f^{\prime}} k_{r e c, c i}^{d k}-n_{c i} k_{c i, d i s s}^{d k}\right) .
\end{gathered}
$$

Here the rate coefficients are introduced for the energy exchange:

$$
A_{c i}+A_{d k} \rightleftharpoons A_{c i^{\prime}}+A_{d k^{\prime}}
$$

exchange chemical reactions:

$$
A_{c i}+A_{d k} \rightleftharpoons A_{c^{\prime} i^{\prime}}+A_{d^{\prime} k^{\prime}}
$$

and dissociation-recombination reactions:

$$
A_{c i}+A_{d k} \rightleftharpoons A_{c^{\prime}}+A_{f^{\prime}}+A_{d k} .
$$


The rate coefficients for the forward reactions (62)-(64) (for collisions of particles $A_{c i}$ and $A_{d k}$ ) are introduced, respectively, as $k_{c, i i^{\prime}}^{d, k k^{\prime}}, k_{c i, c^{\prime} i^{\prime}}^{d k, k^{\prime}}$, and $k_{c i, d i s s^{\prime}}^{d k}$ the recombination rate coefficient is denoted as $k_{r e c, c i}^{d k}$. Note that if $k^{\prime}=k$, then Eq. (62) describes VT(TV) transitions for a molecule $A_{c i}$ during the collision with a molecule $A_{d k}$ with the rate coefficient $k_{c, i i^{\prime}}^{d k}$ of the forward transition. If $d$ is an atom, then the corresponding rate coefficient of the forward transition (62) is $k_{c, i i^{\prime}}^{d}$.

If $k \neq k^{\prime}$ then the reaction (62) describes either $\mathrm{VV}_{1}$ exchange of the vibrational energy between molecules of the same chemical species (for $c=d$ ) or $\mathrm{VV}_{2}$ transitions between molecules of different chemical species (for $c \neq d$ ). Note that $\mathrm{VV}_{1}$ and $\mathrm{VV}_{2}$ transitions of the vibrational energy are almost always accompanied with the transfer of the part of vibrational energy into the translational or rotational modes. However, the probability of a simultaneous exchange between three and more energy modes during one collision is rather low, consequently, these exchanges are usually omitted in the production terms of the kinetic equations.

In the dissociation and recombination reactions (64), the particle $A_{d k}$ can also be either a molecule or an atom. Therefore, different dissociation rate coefficients should be introduced: $k_{r e c, c i}^{d k}, k_{r e c, c i}^{d}$. The rate coefficients of the above processes depend on the order of the distribution function approximation.

The Chapman-Enskog method generalized for the conditions (51) gives the possibility to express, in any approximation, the transport and relaxation terms in Eqs. (57), (11), (12) as functions of the main macroscopic parameters $n_{c i}(\mathbf{r}, t), \mathbf{v}(\mathbf{r}, t)$, and $T(\mathbf{r}, t)$ and thus to close completely the set of governing equations.

In the zero-order approximation (54), (55),

$$
\boldsymbol{P}^{(0)}=n k T \boldsymbol{I}, \quad \mathbf{q}^{(0)}=0, \quad \mathbf{V}_{c i}^{(0)}=0 \quad \forall c, i,
$$

and the governing equations contain the equations of state-to-state kinetics

$$
\frac{d n_{c i}}{d t}+n_{c i} \nabla \cdot \mathbf{v}=R_{c i}^{(0)}, \quad c=1, \ldots, L, \quad i=0, \ldots, L_{c},
$$

coupled to the conservation equations in the form (27), (28).

The right hand sides of Eqs. (66) $R_{c i}^{(0)}$ are specified by the zero-order distribution function. The expressions for $R_{c i}$ contain the microscopic rate coefficients for vibrational energy exchanges and chemical reactions. The equations (66) describe detailed state-to-state vibrational and chemical kinetics in an inviscid non-conductive gas mixture flow in the Euler approximation. In the first-order approximation state-dependent transport properties and reaction rates in reacting non-equilibrium flows are studied in Kustova \& Nagnibeda (1998); Kustova et al. (1999); Nagnibeda \& Kustova (2009).

\subsection{State dependent reaction rate coefficients}

Let us consider state dependent rate coefficients for chemical reactions appearing in Eqs. (59)-(61). In the zero-order Chapman-Enskog approximation rate coefficients for 
exchange reactions have the form Nagnibeda \& Kustova (2009):

$$
\begin{aligned}
k_{c i, c^{\prime} i^{\prime}}^{d k, d^{\prime} k^{\prime}} & =\frac{4 \pi}{Z_{c i}^{r o t} Z_{d k}^{r o t}}\left(\frac{m_{c d}}{2 \pi k T}\right)^{3 / 2} \sum_{j l j^{\prime} l^{\prime}} \int \exp \left(-\frac{m_{c d} g^{2}}{2 k T}\right) \times \\
& \times s_{j}^{c i} s_{l}^{d k} \exp \left(-\frac{\varepsilon_{j}^{c i}+\varepsilon_{l}^{d k}}{k T}\right) g^{3} \tilde{\sigma}_{c d, i j k l}^{c^{\prime} d^{\prime}, i^{\prime} j^{\prime} k^{\prime} l^{\prime}} d g,
\end{aligned}
$$

with $\tilde{\sigma}_{c d, i j k l}^{c^{\prime} d^{\prime}, i^{\prime} j^{\prime} k^{\prime} l^{\prime}}$ designating the integral cross section of a collision resulting in a bimolecular reaction. The integral cross section is obtained integrating the corresponding differential cross sections over solid angles in which relative velocity appear before and after collision:

$$
\tilde{\sigma}_{c d, i j k l}^{c^{\prime} d^{\prime}, i^{\prime} j^{\prime} k^{\prime} l^{\prime}}(g)=\frac{1}{4 \pi} \int \sigma_{c d, i j k l}^{c^{\prime} d^{\prime}, i^{\prime} j^{\prime} k^{\prime} l^{\prime}}(\mathbf{g}, \Omega) d^{2} \Omega d^{2} \Omega^{\prime} .
$$

It is commonly supposed that the cross section depends on the absolute value $g$ of the relative velocity rather than the vector $\mathrm{g}$. Then

$$
\tilde{\sigma}_{c d, i j k l}^{c^{\prime} d^{\prime}, i^{\prime} j^{\prime} k^{\prime} l^{\prime}}(g)=\int \sigma_{c d, i j k l}^{c^{\prime} d^{\prime}, i^{\prime} j^{\prime} k^{\prime} l^{\prime}}(g, \Omega) d^{2} \Omega .
$$

The recombination rate coefficients in the zero-order approximation can be represented in the form

$$
\begin{aligned}
& k_{r e c, c i}^{d k}=\frac{\left(m_{c^{\prime}} m_{f^{\prime}} m_{d}\right)^{3 / 2}}{(2 \pi k T)^{9 / 2}} \sum_{j} \int \sigma_{c^{\prime} f^{\prime} d}^{r e c, c i j}\left(\mathbf{u}_{c^{\prime}}, \mathbf{u}_{f^{\prime}}, \mathbf{u}_{d}^{\prime}, \mathbf{u}_{c}, \mathbf{u}_{d}\right) \times \\
& \times \exp \left(-\frac{m_{c^{\prime}} u_{c^{\prime}}^{2}+m_{f^{\prime}} u_{f^{\prime}}^{2}+m_{d} u_{d}^{\prime 2}}{2 k T}\right) d \mathbf{u}_{c^{\prime}} d \mathbf{u}_{f^{\prime}} d \mathbf{u}_{d}^{\prime} d \mathbf{u}_{c} d \mathbf{u}_{d} .
\end{aligned}
$$

The zero-order dissociation rate coefficients take the form Kuščer (1991); Ludwig \& Heil (1960)

$$
k_{c i, d i s s}^{d k}=\frac{4 \pi}{Z_{c i}^{r o t}}\left(\frac{m_{c d}}{2 \pi k T}\right)^{3 / 2} \sum_{j} \int \exp \left(-\frac{m_{c d} g^{2}}{2 k T}\right) s_{j}^{c i} \exp \left(-\frac{\varepsilon_{j}^{c i}}{k T}\right) g^{3} \tilde{\sigma}_{c i j, d}^{d i s s} d g,
$$

the integral dissociation reaction cross section is introduced by the formula

$$
\tilde{\sigma}_{c i j, d}^{d i s s}=\int \sigma_{c i j, d}^{d i s s}\left(g, \mathbf{u}_{c^{\prime}}, \mathbf{u}_{f^{\prime}}, \mathbf{u}_{d}^{\prime}\right) d \mathbf{u}_{c^{\prime}} d \mathbf{u}_{f^{\prime}} d \mathbf{u}_{d}^{\prime} .
$$

Since it is supposed that the cross sections of dissociation $\sigma_{c i j, d}^{d i s s}$ and recombination $\sigma_{c^{\prime} f^{\prime} d}^{r e c, c i j}$ do not depend on the vibrational state $k$ of the partner $A_{d k}$ in the reaction (64), then:

$$
k_{c i, d i s s}^{d k}=k_{c i, d i s s}^{d} \quad k_{r e c, c i}^{d k}=k_{r e c, c i}^{d}
$$

The relations connecting the rate coefficients of forward and backward collisional processes follow from the microscopic detailed balance relations for reactive collisions (8)-(9) after averaging them with the Maxwell-Boltzmann distribution over the velocity and rotational energy. Thus for the rate coefficients of forward and backward reactions we obtain

$$
K_{c^{\prime} i^{\prime}, c i}^{d^{\prime} k^{\prime}, d k}=\frac{k_{c^{\prime} i^{\prime}, c i}^{d^{\prime} k^{\prime}, d k}}{k_{c i, c^{\prime} i^{\prime}}^{d k, d^{\prime} k^{\prime}}}=\frac{s_{i}^{c} s_{k}^{d}}{s_{i^{\prime}}^{c^{\prime}} s_{k^{\prime}}^{d^{\prime}}}\left(\frac{m_{c} m_{d}}{m_{c^{\prime}} m_{d^{\prime}}}\right)^{3 / 2} \frac{Z_{c i}^{r o t} Z_{d k}^{r o t}}{Z_{c^{\prime} i^{\prime}}^{r o t} Z_{d^{\prime} k^{\prime}}^{r o t}} \times
$$




$$
\begin{gathered}
\times \exp \left(\frac{\varepsilon_{i^{\prime}}^{c^{\prime}}+\varepsilon_{k^{\prime}}^{d^{\prime}}-\varepsilon_{i}^{c}-\varepsilon_{k}^{d}}{k T}\right) \exp \left(\frac{D_{c}+D_{d}-D_{c^{\prime}}-D_{d^{\prime}}}{k T}\right), \\
K_{r e c-d i s s, c i}^{d}=\frac{k_{r e c, c i}^{d}}{k_{c i, d i s s}^{d}}=s_{i}^{c}\left(\frac{m_{c}}{m_{c^{\prime}} m_{f^{\prime}}}\right)^{3 / 2} h^{3}(2 \pi k T)^{-3 / 2} Z_{c i}^{r o t} \exp \left(-\frac{\varepsilon_{i}^{c}-D_{c}}{k T}\right) .
\end{gathered}
$$

In the last formula, $m_{c}=m_{c^{\prime}}+m_{f^{\prime}}$.

For diatomic gases, the vibrational statistical weight $s_{i}^{c}=1$. Moreover, for the rigid rotator model, the rotational partition function is independent of the vibrational state $Z_{c i}^{r o t}=Z_{c}^{r o t}$. In this case, the ratio of the backward and forward reaction rate coefficients takes the reduced form. The expression (74) can be simplified if the collision partner is an atom.

For the application of Egs. (66), (27), (28) to particular problems of non-equilibrium fluid dynamics, the analytical expressions for the dependence of the reaction rate coefficients on the vibrational states of molecules participating in the reactions are needed.

As for vibrational energy transitions, a number of theoretical and experimental estimates for rate coefficients of these transitions are available in the literature in different temperature intervals. These data can be found, for example, in Nagnibeda \& Kustova (2009); Phys-Chem (2002; 2004). The comparison of rate coefficients for vibational energy transitions of $\mathrm{N}_{2}$ molecules obtained using different models is given in Nagnibeda \& Kustova (2009).

The rate coefficients for dissociation from different vibrational levels have been studied much less widely than for vibrational energy transitions. Two models are commonly used for calculations: the ladder-climbing model assuming dissociation only from the last vibrational level (see, for instance, Armenise et al. (1996; 1995); Capitelli et al. (1997); Osipov (1966)), and that of Treanor and Marrone Marrone \& Treanor (1963) allowing for dissociation from any vibrational state.

In the frame of ladder climbing model, the rate of dissociation is specified by the number of molecules occurring on the last vibrational level. Consequently, the dissociation rate is totally specified by the probabilities for the vibrational energy transitions to the last level. In the case when dissociation can occur from any vibrational level, the expression for the rate coefficient for dissociation of a molecule on the vibrational level $i$ can be written in the form Nagnibeda \& Kustova (2009):

$$
k_{i, d i s s}^{d}=Z_{i}^{d}(T) k_{\text {diss }, e q}^{d}(T) .
$$

Here, $k_{\text {diss }, e q}^{d}(T)$ is the thermal equilibrium dissociation rate coefficient obtained by averaging the state-dependent rate coefficient with the one-temperature Boltzmann distribution; $Z_{i}^{d}$ is the non-equilibrium factor. Using the Treanor-Marrone model Marrone \& Treanor (1963), the expression for $Z_{i}^{d}(T)$ was obtained in Nagnibeda \& Kustova (2009):

$$
Z_{i}^{d}(T)=Z_{i}(T, U)=\frac{Z^{\operatorname{vibr}}(T)}{Z^{\operatorname{vibr}}(-U)} \exp \left[\frac{\varepsilon_{i}}{k}\left(\frac{1}{T}+\frac{1}{U}\right)\right],
$$

$Z_{v i b r}$ is the equilibrium vibrational partition function

$$
Z^{v i b r}(T)=\sum_{i} s_{i}^{c} \exp \left(-\frac{\varepsilon_{i}}{k T}\right)
$$


$U$ is a parameter of the model.

Thus, the state-dependent dissociation rate coefficient $k_{i \text {,diss }}^{d}$ is expressed in terms of the averaged thermal equilibrium coefficient $k_{\text {diss,eq }}^{d}(T)$ and non-equilibrium factor (77). To calculate $k_{\text {diss, eq }}^{d}(T)$, the empirical Arrhenius law can be applied:

$$
k_{\text {diss, eq }}^{d}=A T^{n} \exp \left(-\frac{D}{k T}\right),
$$

the coefficients $A$ and $n$ are generally obtained as a best fit to experimental data. The tables of the coefficients in the Arrhenius formula for various chemical reactions can be found in Refs. Gardiner (1984); Kondratiev \& Nikitin (1974); Park (1990); Phys-Chem (2002); Stupochenko et al. (1967).

In Ref. Esposito, Capitelli, Kustova \& Nagnibeda (2000), the dissociation rate coefficients $k_{i, d i s s}^{d}$ calculated within the framework of the Treanor-Marrone model are compared with those obtained from trajectory calculations Esposito, Capitelli \& Gorse (2000), some recommendations for the optimum choice for the parameter $U$ for the specific reactions are given. Figure 1 presents the temperature dependence of the state-dependent dissociation rate coefficients $k_{i \text {, diss }}^{\mathrm{N}_{2}}$ in an $\left(\mathrm{N}_{2}, \mathrm{~N}\right)$ mixture. The coefficients are calculated for different values of the parameter $U$ for two vibrational quantum numbers: $i=0$ and $i=20$. The results of trajectory calculation for $k_{i, \text { diss }}$ taken from Ref. Esposito, Capitelli \& Gorse (2000) are also plotted. We can see that for low vibrational levels, the choice for $U=\infty$ results in significant overestimation for $k_{i, \text { diss }}$, which confirms the common assumption of the preferential dissociation from high vibrational states.

With the increase in the vibrational quantum number, for $U=\infty$ we obtain more realistic values for $k_{i, \text { diss, }}$ and for $i>40$, we have the best agreement with the results of accurate trajectory calculations. $U=D /(6 k)$ and $U=3 T$ provide good consistency for $k_{i, \text { diss }}$ at intermediate levels $(20<i<40)$. Furthermore, $U=D /(6 k)$ results in better consistency for low temperatures, whereas $U=3 T$ is good in the high temperature range $(T>6000 \mathrm{~K})$.
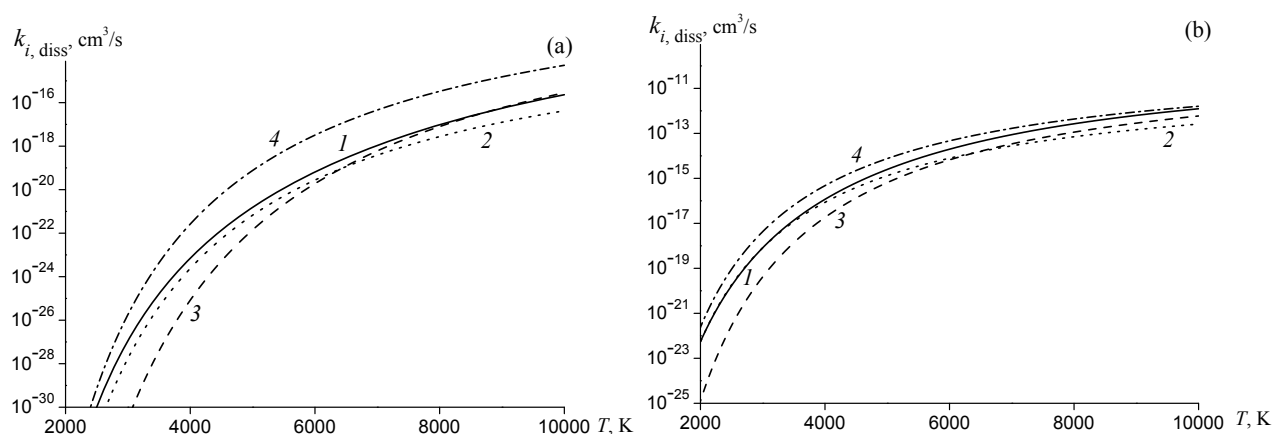

Fig. 1. The temperature dependence of the dissociation rate coefficient $k_{i \text {, diss }}^{\mathrm{N}_{2}}$ for $i=0(a)$ and $i=20(b)$. The curve 1 represents the results obtained in Ref. Esposito, Capitelli \& Gorse (2000), curves $2-4$ correspond to $U=D /(6 k), U=3 T$, and $U=\infty$.

It should be emphasized that using the same value for the parameter $U$ for any $i$ and $T$ can result in considerable errors in the calculation for the state-to-state dissociation rate 
coefficients. The choice for the parameter should be specified by the conditions of a particular problem (the temperature range, basic channels of dissociation, etc.). In some studies Armenise et al. (1996); Candler et al. (1997); Capitelli et al. (1997), a possibility for dissociation from any vibrational state is suggested within the framework of the ladder-climbing model. To this end, it is supposed that a transition to the continuum occurs as a result of multi-quantum vibrational energy transfers.

The rate coefficients for bimolecular exchange reactions depending on the vibrational states of reagents and products have been less thoroughly studied than those for dissociation processes. Theoretical and experimental studies for the influence of the vibrational excitation of reagents on reaction rates were started by J. Polanyi Polanyi (1972); some experimental results were also obtained in Birely \& Lyman (1975). The accurate theoretical approach to this problem primarily requires a calculation for the state-dependent differential cross sections for collisions resulting in chemical reactions, and their subsequent averaging over the velocity distributions. In the recent years, the dynamics of atmospheric reactions has been studied, and quasi-classical trajectory calculations for the cross sections and state-dependent rate coefficients for the reactions $\mathrm{N}_{2}(i)+\mathrm{O} \rightarrow \mathrm{NO}+\mathrm{N}$ and $\mathrm{O}_{2}(i)+\mathrm{N} \rightarrow \mathrm{NO}+\mathrm{O}$ have been carried out by several authors Gilibert et al. (1992; 1993).

At present time, two kinds of analytical expressions are available in the literature. The first kind includes analytical approximations for numerical results obtained for particular reactions (see Refs. Bose \& Candler (1996); Capitelli et al. (1997; 2000); Phys-Chem (2002)). These expressions are sufficiently accurate and convenient for practical use; however, their application is restricted by the considered temperature range. Another approach is based on the generalizations of the Treanor-Marrone model to exchange reactions suggested in Refs. Aliat (2008); Knab (1996); Knab et al. (1995); Seror et al. (1997). These models can be used for more general cases, but the theoretical expressions for the rate coefficients contain additional parameters, which should be validated using experimental data. A lack of the data for these parameters restricts the implementation of the above semi-empirical models.

Therefore, the development of justified theoretical models for cross sections of reactive collisions and state-dependent rate coefficients for exchange reactions remains a very important problem of the non-equilibrium kinetics.

\section{Multi-temperature models for vibrational-chemical kinetics}

\subsection{Governing equations}

The approach proposed in the previous section makes it possible to develop the most rigorous model of reacting gas mixtures, since it takes into account the detailed state-to-state vibrational and chemical kinetics in a flow. However, practical implementation of this method leads to serious difficulties. The first important problem encountered in the realization of the state-to-state model is its computational cost. Indeed, the solution of the fluid dynamics equations coupled to the equations of the state-to-state vibrational and chemical kinetics requires numerical simulation of a great number of equations for the vibrational level populations of all molecular species. The second fundamental problem is that experimental and theoretical data on the state-specific rate coefficients and especially on the cross sections of inelastic processes are rather scanty. Due to the above problems, simpler models based on quasi-stationary vibrational distributions are rather attractive for practical applications. In quasi-stationary approaches, the vibrational level populations are expressed in terms of a 
few macroscopic parameters, consequently, non-equilibrium kinetics can be described by a considerably reduced set of governing equations.

It is known from experiments Gordiets et al. (1988) that in a vibrationally excited gas, near-resonant vibrational energy exchanges between molecules of the same chemical species proceed much faster than non-resonant transitions between different molecules, as well as transfers of vibrational energy to other modes and chemical reactions. Therefore the following relation between the characteristic relaxation times is fulfilled:

$$
\tau_{\text {el }} \lesssim \tau_{\text {rot }}<\tau_{V V_{1}} \ll \tau_{V V_{2}}<\tau_{T R V}<\tau_{\text {react }} \sim \theta,
$$

$\tau_{V V_{1}}$ is the mean time between the collisions of the same species; $\tau_{V V_{2}}$ is the characteristic time of the vibrational energy exchange between different molecules. In the multi-component reacting gas mixture under the condition (80) the integral operators in the kinetic equations (2) includes the operator of $\mathrm{VV}_{1}$ vibrational energy transitions between molecules of the same species along with the operators of elastic collisions and collisions with rotational energy exchanges:

$$
J_{c i j}^{r a p}=J_{c i j}^{e l}+J_{c i j}^{r o t}+J_{c i j}^{V V_{1}} .
$$

The operator of slow processes $J_{c i j}^{s l}$ consists of the operator of $\mathrm{VV}_{2}$ vibrational transitions between molecules of different species, the operator describing the transfer of vibrational energy into rotational and translational modes $J_{c i j}^{T R V}$, as well as the operator of chemical reactions $J_{c i j}^{\text {react }}$ :

$$
J_{c i j}^{s l}=J_{c i j}^{V V_{2}}+J_{c i j}^{T R V}+J_{c i j}^{r e a c t} .
$$

Governing equations of the reacting flows and distribution functions in the zero-order and first-order approximations, under condition (80) are studied in details in Nagnibeda \& Kustova (2009). The distribution function is totally specified by the macroscopic parameters $n_{c}, \mathbf{v}, T$, and $T_{1}^{c}$, where the parameter $T_{1}^{c}$ is the vibrational temperature of the first vibrational level of molecules $c$. The parameter $T_{1}^{c}$ is associated to the additional collision invariant $i_{c}$ which reflects the conservation of the number of vibrational quanta of each molecular species in rapid processes. The zero-order distribution functions in this case may be written in the form (54) where level populations $n_{c i}$ are described by the relation:

$$
n_{c i}=\frac{n_{c}}{Z_{c}^{v i b r}\left(T, T_{1}^{c}\right)} s_{i}^{c} \exp \left(-\frac{\varepsilon_{i}^{c}-i \varepsilon_{1}^{c}}{k T}-\frac{i \varepsilon_{1}^{c}}{k T_{1}^{c}}\right) .
$$

with vibrational partition function

$$
Z_{c}^{v i b r}\left(T, T_{1}^{c}\right)=\sum_{i} s_{i}^{c} \exp \left(-\frac{\varepsilon_{i}^{c}-i \varepsilon_{1}^{c}}{k T}-\frac{i \varepsilon_{1}^{c}}{k T_{1}^{c}}\right) .
$$

$\varepsilon_{1}^{c}$ is the vibrational energy of a molecule $c$ at the first level. Here the vibrational energy is counted from the energy of the zeroth level.

The expression (83) yields the non-equilibrium quasi-stationary Treanor distribution Treanor et al. (1968) generalized for a multi-component reacting gas mixture taking into account anharmonic molecular vibrations and rapid exchange of vibrational quanta. 
The closed set of governing equations for the macroscopic parameters $n_{\mathcal{c}}(\mathbf{r}, t), \mathbf{v}(\mathbf{r}, t)$, $T(\mathbf{r}, t)$, and $T_{1}^{\mathcal{C}}(\mathbf{r}, t)$ derived in Chikhaoui et al. (2000; 1997) includes the equations of the multi-temperature chemical kinetics for the species number densities

$$
\frac{d n_{c}}{d t}+n_{c} \nabla \cdot \mathbf{v}+\nabla \cdot\left(n_{c} \mathbf{V}_{c}\right)=R_{c}^{r e a c t}, c=1, \ldots, L,
$$

relaxation equations for the specific numbers of vibrational quanta $W_{c}$ in each molecular species c:

$$
\rho_{c} \frac{d W_{c}}{d t}+\nabla \cdot \mathbf{q}_{w, c}=R_{c}^{w}-W_{c} m_{c} R_{c}^{r e a c t}+W_{c} \nabla \cdot\left(\rho_{c} \mathbf{V}_{c}\right), \quad c=1, \ldots, L_{\mathrm{m}} .
$$

along with the conservation equations for the momentum and the total energy, $L_{m}$ is the number of molecular species in the mixture. The latter equations formally coincide with the corresponding equations (11) and (12) obtained in the two previous approaches. One should however bear in mind that in the multi-temperature approach, the total energy is a function of $T, T_{1}^{c}$, and $n_{c}$, and the transport terms are expressed as functions of the same set of macroscopic parameters $T, T_{1}^{c}$, and $n_{c}$.

The source terms in Eqs. (85) are determined by the collision operator of chemical reactions

$$
R_{c}^{\text {react }}=\sum_{i j} \iint_{c i j}^{r e a c t} d \mathbf{u}_{c} .
$$

The production terms in the relaxation equations (86) are expressed as functions of collision operators of all slow processes: $\mathrm{VV}_{2}$ and TRV vibrational energy transfers and chemical reactions,

$$
R_{c}^{w}=\sum_{i j} i \int J_{c i j}^{s l} d \mathbf{u}_{c}=R_{c}^{w, V V_{2}}+R_{c}^{w, T R V}+R_{c}^{w, r e a c t} .
$$

The value $\mathbf{q}_{w, c}$ in Eq. (86) has the physical meaning of the vibrational quanta flux of $c$ molecular species and is introduced on the basis of the additional collision invariant of the most frequent collisions $i_{c}$ :

$$
\mathbf{q}_{w, c}=\sum_{i j} i \int \mathbf{c}_{c} f_{c i j} d \mathbf{u}_{c} .
$$

It is obvious that the system of governing equations in the multi-temperature approach is considerably simpler than the corresponding system in the state-to-state approach, since it contains much fewer equations. In the zero-order approximation of the Chapman-Enskog method, the system of governing equations takes the form typical for inviscid non-conductive flows. In this case equations (85), (86) read:

$$
\begin{aligned}
& \frac{d n_{c}}{d t}+n_{c} \nabla \cdot \mathbf{v}=R_{c}^{\text {react }(0)}, \quad c=1, \ldots, L, \\
& \rho_{c} \frac{d W_{c}}{d t}=R_{c}^{w(0)}-m_{c} W_{c} R_{c}^{\text {react }(0)}, c=1, \ldots, L_{\mathrm{m}} .
\end{aligned}
$$




\subsection{Multi-temperature reaction rate coefficients}

The production terms in Eqs. (89) may be written in the form (13), (14) similar to the equations obtained in the one-temperature approximation. However, the coefficients in these expressions differ from those in the one-temperature approach and contains the multi-temperature rate coefficients $k_{c c^{\prime}}^{d d^{\prime}}$ of the reaction (63) (during a collision of two molecules or a molecule and an atom $\mathrm{A}_{d}$ ), the two-temperature coefficients of dissociation $k_{c, d i s s}^{d}$, and recombination rate coefficients $k_{r e c, c}^{d}$.

In the zero-order approximation, the multi-temperature rate coefficients of exchange and dissociation reactions can be expressed in terms of the state-specific rate coefficients considered in section 3.2:

$$
\begin{gathered}
k_{c c^{\prime}}^{d d^{\prime}(0)}=\frac{1}{n_{c} n_{d}} \sum_{i k i^{\prime} k^{\prime}} n_{c i} n_{d k} k_{c i, c^{\prime} i^{\prime}}^{d k, d^{\prime} k^{\prime}(0)}(T), \\
k_{c, \text { diss }}^{d(0)}=\frac{1}{n_{c}} \sum_{i} n_{c i} k_{c i, d i s s}^{d(0)}(T),
\end{gathered}
$$

where $n_{c i}$ denotes some non-equilibrium quasi-stationary distribution.

For the generalized Treanor distribution (83), the multi-temperature rate coefficients of exchange reactions occurring as a result of collisions of two molecules take the form

$$
\begin{gathered}
k_{c c^{\prime}}^{d d^{\prime}(0)}\left(T, T_{1}^{c}, T_{1}^{d}\right)=\frac{1}{Z_{c}^{v i b r}\left(T, T_{1}^{c}\right) Z_{d}^{v i b r}\left(T, T_{1}^{d}\right)} \sum_{i k i^{\prime} k^{\prime}} s_{i}^{c} s_{k}^{d} \exp \left(-\frac{\varepsilon_{i}^{c}-i_{c} \varepsilon_{1}^{c}}{k T}-\right. \\
\left.-\frac{\varepsilon_{k}^{d}-k_{d} \varepsilon_{1}^{d}}{k T}-\frac{i_{c} \varepsilon_{1}^{c}}{k T_{1}^{c}}-\frac{k_{d} \varepsilon_{1}^{d}}{k T_{1}^{d}}\right) k_{c i, c^{\prime} i^{\prime}}^{d k, k^{\prime} k^{\prime}(0)}(T) .
\end{gathered}
$$

The rate coefficient for the exchange reaction in a collision of a diatomic molecule and an atom, as well as the dissociation rate coefficient, depends on two temperatures ( $T$ and $T_{1}^{c}$ ):

$$
\begin{aligned}
& k_{c c^{\prime}}^{d d^{\prime}(0)}\left(T, T_{1}^{c}\right)=\frac{1}{Z_{c}^{v i b r}\left(T, T_{1}^{c}\right)} \sum_{i i^{\prime}} s_{i}^{c} \exp \left(-\frac{\varepsilon_{i}^{c}-i_{c} \varepsilon_{1}^{c}}{k T}-\frac{i_{c} \varepsilon_{1}^{c}}{k T_{1}^{c}}\right) k_{c i, c^{\prime} i^{\prime}}^{d d^{\prime}(0)}(T), \\
& k_{c, \text { diss }}^{d(0)}\left(T, T_{1}^{c}\right)=\frac{1}{Z_{c}^{v i b r}\left(T, T_{1}^{c}\right)} \sum_{i} s_{i}^{c} \exp \left(-\frac{\varepsilon_{i}^{c}-i_{c} \varepsilon_{1}^{c}}{k T}-\frac{i_{c} \varepsilon_{1}^{c}}{k T_{1}^{c}}\right) k_{c i, d i s s}^{d(0)}(T) .
\end{aligned}
$$

The total recombination rate coefficient $k_{\text {rec, }, ~}^{d(0)}$ is defined in terms of the state-specific rate coefficients as follows

$$
k_{\mathrm{rec}, c}^{d(0)}(T)=\sum_{i} k_{\mathrm{rec}, c i}^{d(0)}(T)
$$

and depends on the gas temperature $T$ only. One should keep in mind that the superscript " 0 " in the notations for the state-to-state rate coefficients indicates that they are calculated by averaging the corresponding inelastic collision cross sections with the Maxwell-Boltzmann distribution over the velocity and rotational energy. The relations connecting the multi-temperature rate coefficients of forward and backward reactions can be 
obtained applying the microscopic detailed balance relations for the collision cross sections. For bimolecular reactions we find

$$
\begin{gathered}
k_{c^{\prime} c}^{d^{\prime} d(0)}\left(T, T_{1}^{c^{\prime}}, T_{1}^{d^{\prime}}\right)=\frac{1}{Z_{c^{\prime}}^{v i b r}\left(T, T_{1}^{c^{\prime}}\right) Z_{d^{\prime}}^{v i b r}\left(T, T_{1}^{d^{\prime}}\right)} \sum_{i k i^{\prime} k^{\prime}} s_{i^{\prime}}^{c^{\prime}} s_{k^{\prime}}^{d^{\prime}} \exp \left(-\frac{i_{c^{\prime}}^{\prime} \varepsilon_{1}^{c^{\prime}}}{k T_{1}^{c^{\prime}}}-\right. \\
\left.-\frac{k_{d^{\prime}}^{\prime} \varepsilon_{1}^{d^{\prime}}}{k T_{1}^{d^{\prime}}}-\frac{\varepsilon_{i^{\prime}}^{c^{\prime}}-i_{c^{\prime}}^{\prime} \varepsilon_{1}^{c^{\prime}}}{k T}-\frac{\varepsilon_{k^{\prime}}^{d^{\prime}}-k_{d^{\prime}}^{\prime} \varepsilon_{1}^{d^{\prime}}}{k T}\right) k_{c i, c^{\prime} i^{\prime}}^{d k, d^{\prime} k^{\prime}(0)} K_{c^{\prime} i^{\prime}, c i}^{d^{\prime} k^{\prime}, d k(0)}(T),
\end{gathered}
$$

whereas for dissociation-recombination we can write

$$
k_{r e c, c}^{d(0)}(T)=\sum_{i} k_{r e c, c i}^{d(0)}(T)=\sum_{i} k_{c i, d i s s}^{d(0)}(T) K_{r e c-d i s s, c i}^{d(0)}(T) .
$$

In these formulae, the ratios for the state-to-state rate coefficients $K_{c^{\prime} i^{\prime}, c i}^{d^{\prime} k^{\prime}, d k(0)}(T), K_{r e c-d i s s, c i}^{d(0)}(T)$ are defined in (74), (75).

In the zero-order approximation, $R_{c}^{w(0)}$ includes the vibrational distributions (83) and the state-to-state rate coefficients of $\mathrm{VV}_{2}, \mathrm{VT}$ vibrational energy transitions and chemical reactions.

If anharmonic effects are neglected then the Boltzmann vibrational distributions with the vibrational temperature $T_{v}^{c}$ are valid, and the multi-temperature rate coefficients of the reactions (93)-(95) take the form

$$
\begin{aligned}
& k_{c c^{\prime}}^{d d^{\prime}(0)}\left(T, T_{v}^{c}, T_{v}^{d}\right)=\frac{1}{Z_{c}^{v i b r}\left(T_{v}^{c}\right) Z_{d}^{v i b r}\left(T_{v}^{d}\right)} \times \\
& \quad \times \sum_{i k i^{\prime} k^{\prime}} s_{i}^{c} s_{k}^{d} \exp \left(-\frac{\varepsilon_{i}^{c}}{k T_{\mathrm{v}}^{c}}-\frac{\varepsilon_{k}^{d}}{k T_{\mathrm{v}}^{d}}\right) k_{c i, c^{\prime} i^{\prime}}^{d k, d^{\prime} k^{\prime}}(0)(T),
\end{aligned}
$$

if $d$ is a molecule,

$$
k_{c c^{\prime}}^{d d^{\prime}(0)}\left(T, T_{\mathrm{v}}^{c}\right)=\frac{1}{Z_{\mathcal{C}}^{v i b r}\left(T_{v}^{c}\right)} \sum_{i i^{\prime}} s_{i}^{c} \exp \left(-\frac{\varepsilon_{i}^{c}}{k T_{\mathrm{V}}^{c}}\right) k_{c i, c^{\prime} i^{\prime}}^{d d^{\prime}(0)}(T),
$$

if $d$ is an atom, and

$$
k_{c, \text { diss }}^{d}\left(T, T_{v}^{c}\right)=\frac{1}{Z_{c}^{v i b r}\left(T_{\mathcal{V}}^{c}\right)} \sum_{i} s_{i}^{c} \exp \left(-\frac{\varepsilon_{i}^{c}}{k T_{\mathrm{v}}^{c}}\right) k_{c i, \operatorname{diss}}^{d(0)}(T) .
$$

For the calculation of two-temperature dissociation rate coefficients in the most studies (see Marrone \& Treanor (1963); Phys-Chem (2002))), the two-temperature dissociation rate coefficient $k_{\text {diss }}^{d}$ is associated with the equilibrium averaged coefficient $k_{d i s s, e q}^{d}$ by introducing the two-temperature non-equilibrium factor $Z\left(T, T_{1}, U\right)$ rather than the state-to-state factor $Z_{i}(T, U)$ :

$$
k_{\text {diss }}^{d}=Z\left(T, T_{1}, U\right) k_{\text {diss }, e q}^{d}(T) .
$$

where

$$
Z\left(T, T_{1}, U\right)=\frac{1}{n_{\mathrm{m}}} \sum_{i} n_{i} Z_{i}(T, U)
$$


Using the Treanor distribution (83) for $n_{i}$, the factor $\mathrm{Z}$ is given by the relation

$$
Z\left(T, T_{1}, U\right)=\frac{Z^{\text {vibr }}(T)}{Z^{v i b r}(-U) Z^{v i b r}\left(T, T_{1}\right)} \sum_{i} s_{i} \exp \left[\frac{i \varepsilon_{1}}{k}\left(\frac{1}{T}-\frac{1}{T_{1}}\right)+\frac{\varepsilon_{i}}{k U}\right] .
$$

For the harmonic oscillator model, the non-equilibrium factor is specified by the vibrational temperature $T_{v}$ and can be calculated using the expression:

$$
Z\left(T, T_{v}, U\right)=\frac{Z^{v i b r}(T) Z^{v i b r}\left(T_{f}\right)}{Z^{v i b r}(-U) Z^{v i b r}\left(T_{v}\right)},
$$

where the effective temperature $T_{f}$ is defined as

$$
T_{f}=\left(\frac{1}{T_{v}}-\frac{1}{T}-\frac{1}{U}\right)^{-1}
$$

Figure 2 presents the temperature dependence of the non-equilibrium factor $Z\left(T, T_{1}, U\right)$ in nitrogen for fixed vibrational temperature values. The non-equilibrium factor is calculated for both anharmonic (104) and harmonic (105) oscillator models. We can see that for minor deviations from the equilibrium $\left(T_{1} / T \sim 1\right)$, both models yield similar results, whereas for the ratio $T_{1} / T$ essentially different from unity, the values of $Z$ for harmonic and anharmonic oscillators differ considerably. In particular, for the selected dissociation model, the non-equilibrium factor and hence the dissociation rate coefficient of harmonic oscillators at $T_{1} / T>1$ significantly exceed $Z$ and $k_{\text {diss }}^{d}$, respectively, when calculated for anharmonic oscillators. For $T_{1} / T<1$, the use of the harmonic oscillator model yields lower $Z$ and $k_{d i s s}^{d}$ than those obtained taking into account anharmonic effects.

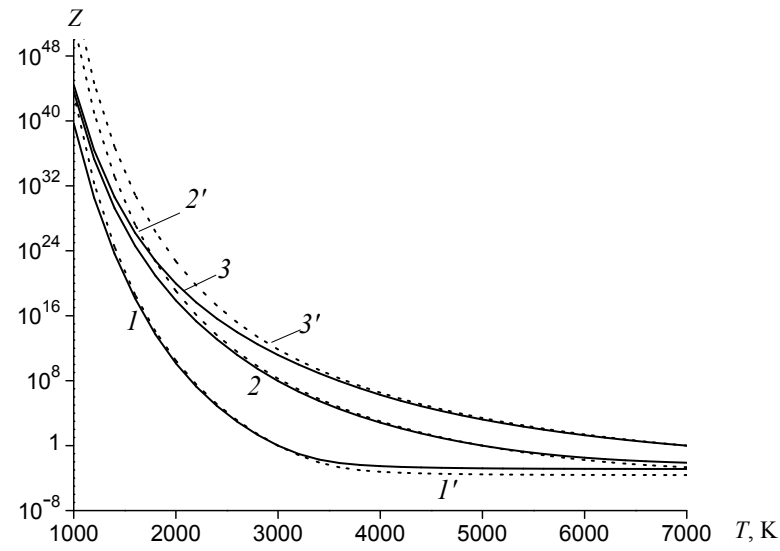

Fig. 2. The non-equilibrium factor $\mathrm{Z}$ in $\mathrm{N}_{2}$ as a function of temperature $T$ for fixed temperatures $T_{1}$ and $U=D /(6 k)$. The solid lines represent anharmonic oscillators, dashed - harmonic oscillators. The curves $1,1^{\prime}-T_{1}=3000 ; 2,2^{\prime}-T_{1}=5000 ; 3,3^{\prime}-T_{1}=7000 \mathrm{~K}$. 
Note in addition that the ratio of the dissociation and recombination rate coefficients $K_{\text {rec-diss }}$ under the non-equilibrium conditions can also be expressed in terms of the averaged non-equilibrium factor:

$$
K_{\text {rec-diss }}=\frac{k_{r e c}^{d}}{k_{\text {diss }}^{d}}=\frac{1}{Z} K_{r e c-d i s s}^{e q}(T),
$$

where $K_{r e c-d i s s}^{e q}(T)$ is the ratio of the dissociation and recombination rate coefficients in a thermal equilibrium gas. Calculating $Z$ from the state-to-state or quasi-stationary vibrational distributions $n_{i} / n$ for various dissociation models, we can find $K_{\text {rec-diss }}$ under thermal non-equilibrium conditions and estimate its deviation from the equilibrium constant.

The equations of non-equilibrium reacting flows derived in the state-to-state, multi-temperature and one-temperature approaches were applied for calculations of distributions and macroscopic parameters in particular flows of air components behind shock waves, in nozzles, in non-equilibrium boundary layer (see Nagnibeda \& Kustova (2009) and references in this book). On the basis of obtained distributions, global reaction rates (92) were calculated in relaxation zone behind the shock wave Kustova \& Nagnibeda (2000) and in nozzle expansion Kustova et al. (2003) in different approaches. The results obtained for the relaxation zone behind the shock wave at the following free stream conditions: $T_{0}=293 \mathrm{~K}$, $p_{0}=100 \mathrm{~Pa}, M_{0}=15$ are presented in Fig. 3 .

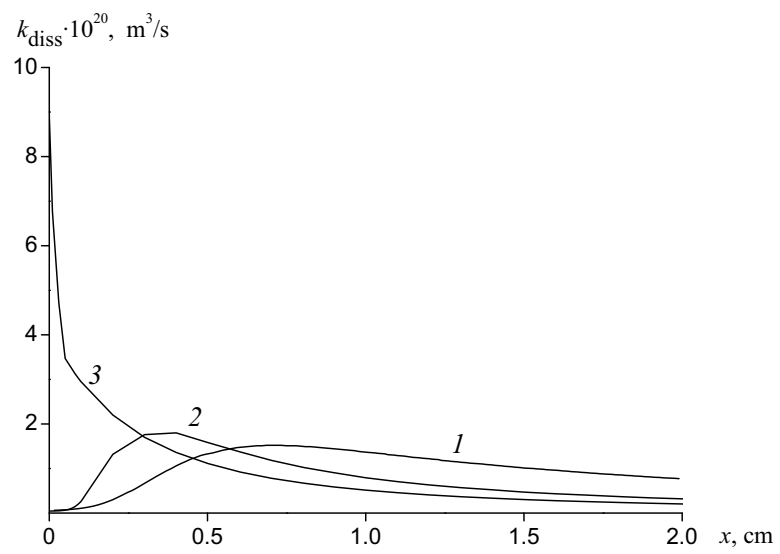

Fig. 3. The averaged dissociation rate coefficient $k_{d i s s}^{N_{2}}$ as a function of $x$. Curves 1, 2, 3 are, respectively, for the state-to-state, two-temperature, and one-temperature approaches.

It is seen that the one-temperature model describes the behavior of the dissociation rate coefficient inadequately, particularly close to the shock front. The two-temperature approach provides more realistic values for the dissociation rate coefficient, overestimating however $k_{\text {diss }}^{N_{2}}$ in comparison to the state-to-state approximation at $x<0.5 \mathrm{~cm}$.

The averaged dissociation rate coefficient $k_{\text {diss }}^{m o l}$ calculated for $\mathrm{O}_{2} / \mathrm{O}$ and $\mathrm{N}_{2} / \mathrm{N}$ mixtures in a conic nozzle in four approaches using state-to-state, multi-temperature and one-temperature distributions, is presented in Fig. 4. The following conditions in the throat are considered: for $\mathrm{O}_{2} / \mathrm{O}$ mixture, $T_{*}=4000 \mathrm{~K}, p_{*}=1 \mathrm{~atm}$; for $\mathrm{N}_{2} / \mathrm{N}$ mixture, $T_{*}=7000 \mathrm{~K}, p_{*}=1 \mathrm{~atm}$. Two kinds of multi-temperature distributions are applied: the Boltzmann distribution for harmonic 

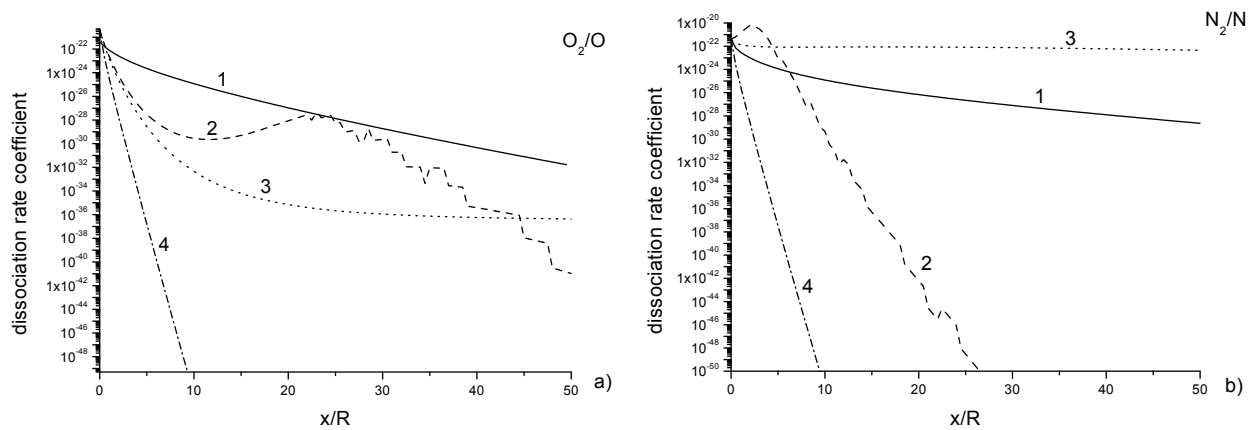

Fig. 4. Averaged dissociation rate coefficient $k_{\text {diss }}^{(m o l)}\left(\mathrm{m}^{3} / \mathrm{s}\right)$ versus $\mathrm{x} / \mathrm{R}$ in a conic nozzle. (a) $\mathrm{O}_{2} / \mathrm{O}, T_{*}=4000 \mathrm{~K}, p_{*}=1 \mathrm{~atm} ;(\mathbf{b}) \mathrm{N}_{2} / \mathrm{N}, T_{*}=7000 \mathrm{~K}, p_{*}=1 \mathrm{~atm}$. Curves 1: state-to-state model; 2: two-temperature anharmonic oscillator model; 3: two-temperature harmonic oscillator model; 4: one-temperature model.

oscillators and a complex distribution for anharmonic oscillators studied in Kustova et al. (2003). One can see a quite strong influence of the kinetic model on the averaged dissociation rate coefficients, all quasi-stationary models give the values of $k_{\text {diss }}^{m o l}$ rather far from those obtained in the rigorous state-to-state approximation, the same effect is obtained for $k_{\text {diss }}^{a t}$. In all considered cases, $k_{\text {diss }}^{a t}$ is higher than $k_{\text {diss }}^{m o l}$, i.e., atoms are more efficient as partners in the dissociation process.

\section{Conclusions}

In this Chapter, the theoretical models for non-equilibrium chemical kinetics in multi-component reacting gas flows are proposed on the basis of three approaches of the kinetic theory. In the frame of the one-temperature approximation the chemical kinetics in thermal equilibrium flows or deviating weakly from thermal equilibrium is studied. The coupling of chemical kinetics and fluid dynamics equations is considered in the Euler and Navier-Stokes approximations. Chemical kinetics in vibrationally non-equilibrium flows is considered on the basis of the state-to-state and multi-temperature approaches. Different models for vibrational-chemical coupling in the flows of multi-component mixtures are derived. The influence of non-equilibrium distributions on reaction rates in the flows behind shock waves and in nozzle expansion is demonstrated.

\section{Acknowledgements}

We are grateful to the Russian Foundation for Basic Research (Grant 11-01-00408) and Ministry of Education and Science of RF (Contract 13.G25.31.0076) for the support of this study.

\section{References}

Alexeev, B., Chikhaoui, A. \& Grushin, I. (1994). Application of the generalized Chapman-Enskog method to the transport-coefficients calculation in a reacting gas mixture, Phys. Review E 49: 2809. 
Alexeev, B. \& Grushin, I. (1994). Transport Processes in Reacting Gases and Plasma, Energoatomizdat, Moscow.

Aliat, A. (2008). State-to-state dissociation-recombination and chemical exchange rate coefficients in excited diatomic gas flows, Physica A 387: 4163-4182.

Armenise, I., Capitelli, M., Colonna, G. \& Gorse, C. (1996). Nonequilibrium vibrational kinetics in the boundary layer of re-entering bodies, J. Thermophys. Heat Transfer 10(3): 397-405.

Armenise, I., Capitelli, M. \& Gorse, C. (1995). On the coupling of non-equilibrium vibrational kinetics and dissociation-recombination processes in the boundary layer surrounding an hypersonic reentry vehicle, in J. Hunt (ed.), Aerothermodynamics for Space Vehicles, ESA Publication Division, ESTEC, Noordwijk, The Netherlands.

Belouaggadia, N. \& Brun, R. (1997). Chemical rate constants in nonequilibrium flows, AIAA Paper 97-2555.

Birely, J. \& Lyman, J. (1975). Effect of reagent vibrational energy on measured reaction rate constants, J. Photochem. 4: 269.

Bose, D. \& Candler, G. (1996). Thermal rate constants of the $\mathrm{N}_{2}+\mathrm{O} \rightarrow \mathrm{NO}+\mathrm{N}$ reaction using $a b$ initio ${ }^{3} A^{\prime \prime}$ and ${ }^{3} A^{\prime}$ potential energy surfaces, J. Chem. Phys. 104(8): 2825.

Candler, G., Olejniczak, J. \& Harrold, B. (1997). Detailed simulation of nitrogen dissociation in stagnation regions, Phys. Fluids 9(7): 2108-2117.

Capitelli, M., Armenise, I. \& Gorse, C. (1997). State-to-state approach in the kinetics of air components under re-entry conditions, J. Thermophys. Heat Transfer 11(4): 570-578.

Capitelli, M., Ferreira, C., Gordiets, B. \& Osipov, A. (2000). Plasma Kinetics in Atmospheric Gases, Vol. 31 of Springer series on atomic, optical and plasma physics, Springer-Verlag, Berlin.

Chikhaoui, A., Dudon, J., Genieys, S., Kustova, E. \& Nagnibeda, E. (2000). Multi-temperature kinetic model for heat transfer in reacting gas mixture, Phys. Fluids 12(1): 220-232.

Chikhaoui, A., Dudon, J., Kustova, E. \& Nagnibeda, E. (1997). Transport properties in reacting mixture of polyatomic gases, Physica A 247(1-4): 526-552.

Ern, A. \& Giovangigli, V. (1994). Multicomponent Transport Algorithms, Lect. Notes Phys., Series monographs, M24, Springer-Verlag.

Ern, A. \& Giovangigli, V. (1998). The kinetic chemical equilibrium regime, Physica $A$ 260: 49-72.

Esposito, F., Capitelli, M. \& Gorse, C. (2000). Quasi-classical dynamics and vibrational kinetics in $N_{2}(v)-N$ system, Chem. Phys. 257: 193-202.

Esposito, F., Capitelli, M., Kustova, E. \& Nagnibeda, E. (2000). Rate coefficients for the reaction $\mathrm{N}_{2}(\mathrm{i})+\mathrm{N}=3 \mathrm{~N}$ : a comparison of trajectory calculations and the Treanor-Marrone model., Chem. Phys. Lett. 330: 207-211.

Ferziger, J. \& Kaper, H. (1972). Mathematical Theory of Transport Processes in Gases, North-Holland, Amsterdam, London.

Gardiner, W. (ed.) (1984). Combustion Chemistry, Springer-Verlag.

Gilibert, M., Aguilar, A., Gonzales, M., Mota, F. \& Sayos, R. (1992). Dynamics of the $N\left({ }^{4} S_{u}\right)+$ $N O\left(X^{2} \Pi\right) \rightarrow N_{2}\left(X^{1} \Sigma_{g}^{+}\right)+O\left({ }^{3} P_{g}\right)$ atmospheric reaction on the ${ }^{3} A^{\prime \prime}$ ground potential energy surface. I. Analytical potential energy surface and preliminary quasiclassical trajectory calculation, J. Chem. Phys. 97: 5542-5552.

Gilibert, M., Aguilar, A., Gonzales, M. \& Sayos, R. (1993b). A quasiclassical trajectory study of the effect of the initial rovibrational level and relative translational energy on the dynamics of the $N\left({ }^{4} S_{u}\right)+\mathrm{O}_{2}\left({ }^{3} \Sigma_{g}^{-}\right) \rightarrow N O\left({ }^{2} \Pi_{u}\right)+O\left({ }^{3} P_{g}\right)$ atmospheric reaction on the ${ }^{2} A^{\prime}$ ground potential energy surface, Chem. Phys. 178: 287-303. 
Gordiets, B., Osipov, A. \& Shelepin, L. (1988). Kinetic Processes in Gases and Molecular Lasers, Gordon and Breach Science Publishers, Amsterdam.

Knab, O. (1996). A physically consistent vibration-chemistry-vibration coupling model, in M. Capitelli (ed.), Molecular Physics and Hypersonic Flows, Kluwer Acad. Publishers, Netherlands, pp. 691-702.

Knab, O., Frühauf, H. \& Messerschmid, E. (1995). Theory and validation of the physically consistent coupled vibration-chemistry-vibration model, J. Thermophys. Heat Transfer 9(2): 219-226.

Kondratiev, V. \& Nikitin, E. (1974). Kinetics and Mechanism of Gas Phase Reactions, Nauka, Moscow.

Kuščer, I. (1991). Dissociation and recombination in an inhomogeneous gas, Physica $A$ 176: 542-556.

Kustova, E. (2009). On the role of bulk viscosity and relaxation pressure in non-equilibrium flows, in T. Abe (ed.), Rarefied Gas Dynamics: 26th International Symposium, Vol. 1084 of AIP Conference Proceedings, pp. 807-812.

Kustova, E. \& Nagnibeda, E. (1998). Transport properties of a reacting gas mixture with strong vibrational and chemical nonequilibrium, Chem. Phys. 233: 57-75.

Kustova, E. \& Nagnibeda, E. (2000). The influence of the state-to-state distributions behind shock wave on the dissociation rates, in G. Ball, R. Hillier \& G. Roberts (eds), Proc. of the 22d International Symposium on Shock Waves, Vol. 1, University of Southhampton, Southhampton, UK. 783-788.

Kustova, E. \& Nagnibeda, E. (2011). Different approaches for modelling of heat transfer in non-equilibrium reacting gas flows, in A. Ahsan (ed.), Evaporation, Condensation and Heat transfer, InTech, ISBN: 978-953-307-583-9, Available from: http://www.intechopen.com/articles/show/title/different-approaches-formodelling-of-heat-transfer-in-non-equilibrium-reacting-gas-flows, pp. 439-464.

Kustova, E., Nagnibeda, E., Alexandrova, T. \& Chikhaoui, A. (2003). Non-equilibrium dissociation rates in expanding flows, Chem. Phys. Lett. 377(5-6): 663-671.

Kustova, E., Nagnibeda, E. \& Chauvin, A. (1999). State-to-state nonequilibrium reaction rates, Chem. Phys. 248(2-3): 221-232.

Kustova, E., Nagnibeda, E. \& Giordano, D. (January 2008). Mutual influence between flow compressibility and chemical-reaction rates in gas mixtures, Esa str-255, ESA Communication Production Office, ESTEC, Noordwijk, The Netherlands.

Ludwig, G. \& Heil, M. (1960). Boundary layer theory with dissociation and ionization, Advances in Applied Mechanics, Vol. VI, Academic Press, New York.

Marrone, P. \& Treanor, C. (1963). Chemical relaxation with preferential dissociation from excited vibrational levels, Phys. Fluids 6(9): 1215.

Nagnibeda, E. \& Kustova, E. (2009). Nonequilibrium Reacting Gas Flows. Kinetic Theory of Transport and Relaxation Processes, Springer-Verlag, Berlin, Heidelberg.

Osipov, A. (1966). Thermal dissociation of diatomic molecules at high temperatures, Teor. Exp. Khim. 2(11): 649.

Park, C. (1990). Nonequilibrium Hypersonic Aerothermodynamics, J.Wiley and Sons, New York, Chichester, Brisbane, Toronto, Singapore.

Phys-Chem (2002). Physical and Chemical Processes in Gas Dynamics: Cross Sections and Rate Constants for Physical and Chemical Processes. Volume I, Vol. I96 of Progress in Astronautics and Aeronautics. 
Phys-Chem (2004). Physical and Chemical Processes in Gas Dynamics: Physical and Chemical Kinetics and Thermodynamics. Volume II, Vol. 197 of Progress in Astronautics and Aeronautics.

Polanyi, J. (1972). Some concepts in reaction dynamics, Acc. Chem. Res. 5: 161-168.

Present, R. (1960). Chapman-Enskog method in chemical kinetics, J. Chem. Phys. 48: 4875-4877.

Prigogine, I. \& Xhrouet, E. (1949). On the perturbation of Maxwell distribution function by chemical reaction in gases, Physica 15: 913-932.

Rydalevskaya, M. (1977). Formal kinetic description of gas mixtures with dissociation and recombination, Aerodinamika Razrezhennykh Gazov, Vol. IX, Leningrad University Press, Leningrad. (in Russian).

Seror, S., Druguet, M., Schall, E. \& Zeitoun, D. (1997). A new vibration-exchange reaction coupling model for hypersonic air flows, AIAA Paper 97-2556.

Shizgal, B. \& Karplus, M. (1970). Nonequilibrium contributions to the rate of reaction. I. Perturbation of the velocity distribution function, J. Chem. Phys. 52: 4262-4278.

Stupochenko, Y., Losev, S. \& Osipov, A. (1967). Relaxation in Shock Waves, Springer-Verlag, Berlin, Heidelberg, New York.

Treanor, C., Rich, I. \& Rehm, R. (1968). Vibrational relaxation of anharmonic oscillators with exchange dominated collisions, J. Chem. Phys. 48: 1798. 


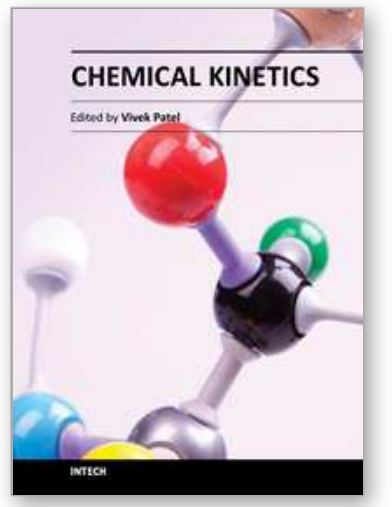

\author{
Chemical Kinetics \\ Edited by Dr Vivek Patel
}

ISBN 978-953-51-0132-1

Hard cover, 344 pages

Publisher InTech

Published online 29, February, 2012

Published in print edition February, 2012

Chemical Kinetics relates to the rates of chemical reactions and factors such as concentration and temperature, which affects the rates of chemical reactions. Such studies are important in providing essential evidence as to the mechanisms of chemical processes. The book is designed to help the reader, particularly students and researchers of physical science, understand the chemical kinetics mechanics and chemical reactions. The selection of topics addressed and the examples, tables and graphs used to illustrate them are governed, to a large extent, by the fact that this book is aimed primarily at physical science (mainly chemistry) technologists. Undoubtedly, this book contains "must read" materials for students, engineers, and researchers working in the chemistry and chemical kinetics area. This book provides valuable insight into the mechanisms and chemical reactions. It is written in concise, self-explanatory and informative manner by a world class scientists in the field.

\title{
How to reference
}

In order to correctly reference this scholarly work, feel free to copy and paste the following:

E. V. Kustova and E. A. Nagnibeda (2012). Vibrational and Chemical Kinetics in Non-Equilibrium Gas Flows, Chemical Kinetics, Dr Vivek Patel (Ed.), ISBN: 978-953-51-0132-1, InTech, Available from: http://www.intechopen.com/books/chemical-kinetics/vibrational-chemical-kinetics-in-non-equilibrium-gas-flows

\section{INTECH}

open science | open minds

\author{
InTech Europe \\ University Campus STeP Ri \\ Slavka Krautzeka 83/A \\ 51000 Rijeka, Croatia \\ Phone: +385 (51) 770447 \\ Fax: +385 (51) 686166 \\ www.intechopen.com
}

\author{
InTech China \\ Unit 405, Office Block, Hotel Equatorial Shanghai \\ No.65, Yan An Road (West), Shanghai, 200040, China \\ 中国上海市延安西路65号上海国际贵都大饭店办公楼 405 单元 \\ Phone: +86-21-62489820 \\ Fax: $+86-21-62489821$
}


(C) 2012 The Author(s). Licensee IntechOpen. This is an open access article distributed under the terms of the Creative Commons Attribution 3.0 License, which permits unrestricted use, distribution, and reproduction in any medium, provided the original work is properly cited. 